\title{
Dynamic Stability of Vortex Solutions of Ginzburg-Landau and Nonlinear Schrödinger Equations
}

\author{
M.I. Weinstein ${ }^{1}$, J. $\mathbf{X i n}^{2}$ \\ ${ }^{1}$ Department of Mathematics, University of Michigan, Ann Arbor, MI 48109, USA \\ ${ }^{2}$ Department of Mathematics, University of Arizona, Tucson, AZ 85721, USA
}

Received: 1 November 1995/Accepted: 15 February 1996

\begin{abstract}
The dynamic stability of vortex solutions to the Ginzburg-Landau and nonlinear Schrödinger equations is the basic assumption of the asymptotic particle plus field description of interacting vortices. For the Ginzburg-Landau dynamics we prove that all vortices are asymptotically nonlinearly stable relative to small radial perturbations. Initially finite energy perturbations of vortices decay to zero in $L^{p}\left(\mathbb{R}^{2}\right)$ spaces with an algebraic rate as time tends to infinity. We also prove that under general (nonradial) perturbations, the plus and minus one-vortices are linearly dynamically stable in $L^{2}$; the linearized operator has spectrum equal to $(-\infty, 0]$ and generates a $C_{0}$ semigroup of contractions on $L^{2}\left(\mathbb{R}^{2}\right)$. The nature of the zero energy point is clarified; it is resonance, a property related to the infinite energy of planar vortices. Our results on the linearized operator are also usẽd to show that the plus and minus one-vortices for the Schrödinger (Hamiltonian) dynamics are spectrally stable, i.e. the linearized operator about these vortices has $\left(L^{2}\right)$ spectrum equal to the imaginary axis. The key ingredients of our analysis are the Nash-Aronson estimates for obtaining Gaussian upper bounds for fundamental solutions of parabolic operators, and a combination of variational and maximum principles.
\end{abstract}

\section{Introduction}

In this paper, we study the dynamic stability of vortex solutions of the GinzburgLandau and nonlinear Schrödinger equations:

$$
\begin{gathered}
u_{t}=\Delta u+\left(1-|u|^{2}\right) u=\frac{\delta \mathscr{E}}{\delta \bar{u}}, \\
-i u_{t}=\Delta u+\left(1-|u|^{2}\right) u=\frac{\delta \mathscr{E}}{\delta \bar{u}} .
\end{gathered}
$$

Here, $u=u(t, x)$ is a complex valued function defined for each $t>0$ and $x=$ $\left(x_{1}, x_{2}\right) \in \mathbb{R}^{2} . \Delta=\partial_{x_{1}}^{2}+\partial_{x_{2}}^{2}$ denotes the two-dimensional Laplacian. The energy 
functional

$$
\mathscr{E}=\int_{R^{2}}\left(\frac{1}{2}|\nabla u|^{2}+\frac{1}{4}\left(|u|^{2}-1\right)^{2}\right) d x .
$$

The Ginzburg-Landau equation arises in the theory of superconductivity; see $[4,11,20]$ and references therein. The nonlinear Schrödinger equation is a basic model for superfluids; see, for example, $[7,10,24,15,12]$. These equations also play a central role as universal envelope equations for bifurcation problems and pattern dynamics; see, for example, [25].

Equations (1.1) and (1.2) admit vortex solutions. These are solutions of the form:

$$
\begin{aligned}
& \Psi_{n}(x)=U_{n}(r) e^{i n \theta}, \quad n= \pm 1, \pm 2, \ldots \\
& U_{n}(0)=0, \quad U_{n}(+\infty)=1
\end{aligned}
$$

where $(r, \theta)$ denote the polar coordinates $\mathbb{R}^{2}$. The functions $\Psi_{n}(x)$ define complex vector fields in the plane: $\left(x_{1}, x_{2}\right) \mapsto\left(\operatorname{Real} \Psi_{n}, \operatorname{Imag} \Psi_{n}\right)$, whose zeros are called vortices or defects. Since the evolution equations (1.1) and (1.2) define continuous deformations of the complex vector field, $u(\cdot, x)$, if the initial total winding number or circulation at infinity is different from zero, one expects a principal feature of the dynamics to be the interaction of vortices or local flow fields organized around the zeros of $u(t, x)$. A description of the dynamics of an ensemble of spatially separated vortices, each having the local structure (1.4), is therefore of fundamental interest.

The systematic study of this problem was initiated by Neu [24]; see also the work of Pismen and Rubinstein [30], and E [11]. In these works, the regime of small $\varepsilon$, the ratio of vortex core size to the separation distance between vortices, is considered. In addition to his asymptotic analysis, Neu [24] presents numerical evidence for the stability of one-vortices and the fission instability of $n$-vortices $(|n| \geqq 2)$. This motivates the underlying assumption of these asymptotic studies that the one-vortices $(|n|=1$ in (1.4)) are stable. For $\varepsilon$ small, a solution is sought in the form of a product of one-vortices plus small error terms of higher order:

$$
u(t, x)=\prod_{i=1}^{N} \Psi_{n_{i}}\left(\frac{x-x_{i}(t, \varepsilon)}{\varepsilon}\right)+O(\varepsilon),
$$

where $n_{i}= \pm 1, N \geqq 2$. Since, $U_{n}(r) \rightarrow 1$ as $r \rightarrow \infty$, the ansatz (1.5) incorporates the assumption that for $x$ in a neighborhood of $x_{j}(t, \varepsilon), u(t, x) \sim \Psi_{n_{j}}\left(\frac{x-x_{j}(t, \varepsilon)}{\varepsilon}\right)$.

In the small $\varepsilon$ limit, matched asymptotic analysis is used to derive a coupled system of ordinary differential equations for the functions $x_{i}(t), i=1, \ldots, N$, which describe the centers of the widely separated vortices. In the Ginzburg-Landau case, the motion of the vortex centers is governed by gradient flow dynamics, while in the Schrödinger case, by Kirchhoff's equations for point vortices of ideal incompressible Euler equation; see [12] for another formal derivation.

An alternative approach is to rescale (1.1) and (1.2) by $X=\varepsilon x, T=\varepsilon^{2} t$. The rescaled equations are the same except that the factor $\varepsilon^{-2}$ appears in front of nonlinearities. The problem then is to take the singular limit $\varepsilon \rightarrow 0$. In recent work, F.-H. Lin $([20,21])$ proved the validity of the motion law of vortices in the rescaled Ginzburg-Landau equation on a bounded domain with Dirichlet boundary data (see 
also [31] for related results). The main tool is energy comparison based on the energy functionals and the characterization of their minimizers in the limit $\varepsilon \rightarrow 0$ studied earlier in Bethuel, Brézis and Helein [4] for static vortices.

Regarding stability, there is work on the Ginzburg-Landau equation considered on the unit disc. Lieb and Loss [19] showed that $\mathscr{E}$, restricted to functions satisfying certain symmetry assumptions, has nonnegative second variational derivative at $n= \pm 1$ vortices. More recently, Mironescu [22] further showed that the second variational derivative at the $n= \pm 1$ vortices is positive definite, and hence the spectrum of the linearized operator is strictly positive. This result can be recovered using our method. See Theorem 5.2 for a nonlinear asymptotic stability result in this case.

For the case of the entire plane, $\mathbb{R}^{2}$, it remains an open problem to prove the validity of the effective particle description of interacting vortices on long time scales. A principal difficulty is that vortex solutions have infinite energy (see (1.3)) and are therefore difficult to treat by variational methods. (A construction of the vortices as minimizers of a relative or renormalized energy was given in [34].) For the Ginzburg-Landau equation (1.1), Bauman, Chen, Phillips, and Sternberg [3] proved the large time asymptotic convergence of a class of solutions with zero winding number to the finite energy steady states consisting of constants of modulus one $|u|=1$. The vortex solutions of the gradient flow generated by the Abelian Higgs functional in the case of critical coupling turn out to have finite energy [16]. Demoulini and Stuart [9] showed the convergence of each solution to a unique static vortex solution of the same winding number.

Our goal of this paper is to investigate the stability properties of the vortex solutions (1.4) under finite energy or $L^{2}\left(\mathbb{R}^{2}\right)$ perturbations. We confirm the basic assumption of the interacting particle plus field description of interacting vortices concerning the stability of one-vortices. We view this as a step toward providing a rigorous description of the motion of well-separated vortices on the plane.

Our main results are:

Theorem 1.1 (Ginzburg-Landau Vortices). Consider (1.1) with initial data:

$$
u_{0}(0, r, \theta)=\Psi_{n}+v_{0}(r, \theta) e^{i n \theta}, \quad n= \pm 1, \pm 2, \ldots,
$$

where $v_{0}(r, \theta)$ is a general complex valued function. We decompose solutions of the initial value problem as:

$$
u(t, r, \theta)=\Psi_{n}+v(t, r, \theta) e^{i n \theta}
$$

and $v$ satisfies the evolution equation:

$$
\left(\begin{array}{l}
v \\
\bar{v}
\end{array}\right)_{t}=M\left(\begin{array}{l}
v \\
\bar{v}
\end{array}\right)+N\left(\begin{array}{l}
v \\
\bar{v}
\end{array}\right),
$$

where $M$ is the self-adjoint linearized operator and $N(\cdot)$ consists of nonlinear terms. Then:

1) Nonlinear asymptotic stability for radial data. If $v_{0}=v_{0}(r) \in L^{p} \cap L^{q}\left(\mathbb{R}^{2}\right)$, where $p \in[3,6), q=\gamma^{-1} p, \gamma \in\left(1+\frac{p}{3}, 3\right)$, there exists an $\varepsilon=\varepsilon\left(p, \gamma, \Psi_{n}\right)>0$ such that as long as $\left\|v_{0}\right\|_{L^{p} \cap L^{q}} \leqq \varepsilon, E q$. (1.6) has unique global mild solution 
$v \in C\left([0, \infty) ; L^{p}\right)$. Moreover,

$$
\|v(t)\|_{L^{p}} \leqq C(1+t)^{-(\gamma-1) p^{-1}},
$$

for $t \geqq 0$ with positive constant $C=C(\varepsilon)$.

2) Linear dynamic stability for general data. If $v_{0}=v_{0}(r, \theta) \in L^{2}\left(\mathbb{R}^{2}\right)$ and $|n|=1$, solution $v=v(r, \theta, t)$ of the linearized equation $v_{t}=M v$ satisfies:

$$
\|v(t)\|_{L^{2}} \leqq\left\|v_{0}\right\|_{L^{2}}, \quad \forall t \geqq 0 .
$$

3) Resonance at zero energy. The $L^{2}$ spectrum of the operator, $M$, is $(-\infty, 0]$. Associated to the zero point in the spectrum is a resonance mode, generated by translation invariance of (1.1). This function does not lie in $L^{2}$ but lies in $L^{p}$ for any $p>2$.

4) Nonlinear asymptotic stability of vortices on $|x| \leqq R$. The $n= \pm 1$ vortices, $e^{i n \theta} U_{R}(r)$, defined on the disc of radius $R, B_{R}$, are nonlinearly exponentially asymptotically stable relative to all small $L^{2}$ perturbations which vanish on the boundary of $B_{R}$.

Remark. The result concerning the zero energy resonance has implications for the behavior of the resolvent, $(M-\lambda I)^{-1}$ as $\lambda \rightarrow 0$, and therefore the time-decay properties as $t \rightarrow \infty$ of the linearized evolution in suitable function spaces [17]. The resulting slower time decay is a subtlety which would have impact on a nonlinear stability theory of vortices in the plane.

Theorem 1.2 (Spectral Stability of Schrödinger Vortices). Consider (1.2) with initial data:

$$
u_{0}(0, r, \theta)=\Psi_{n}+v_{0}(r, \theta) e^{i n \theta}, \quad n= \pm 1,
$$

where $v_{0}(r, \theta)$ is a complex valued function. We decompose solutions of the initial value problem as:

$$
u(t, r, \theta)=\Psi_{n}+v(t, r, \theta) e^{i n \theta},
$$

where $v$ satisfies the evolution equation:

$$
\left(\begin{array}{c}
v \\
\bar{v}
\end{array}\right)_{t}=i \sigma M\left(\begin{array}{c}
v \\
\bar{v}
\end{array}\right)+N\left(\begin{array}{c}
v \\
\bar{v}
\end{array}\right) .
$$

Here i $\sigma M$ denotes the linearized operator, $N(\cdot)$ is the nonlinear part, and

$$
\sigma=\left(\begin{array}{rr}
1 & 0 \\
0 & -1
\end{array}\right)
$$

Then the $L^{2}$ spectrum of $i \sigma M$ is equal to the imaginary axis.

The remainder of the paper is organized as follows. In Sect. 2, we derive Eq. (1.6) for perturbation $v$ and write the equation as a coupled parabolic system for the real and imaginary parts of $v$. Sections 3 through 5 address the dynamics of vortices in the context of the Ginzburg-Landau equations, with Sects. 3 and 4 focusing on the radial case. In Sect. 3, we employ the vortex profile equation to convert the linearized operators into divergence form. Due to the vanishing of the vortex 
profile at $r=0$, the parabolic operator of divergence form is degenerate at zero. We adapt the classical Nash-Aronson estimates using cutoff functions to obtain a pointwise Gaussian upper bound for the fundamental solutions. In Sect. 4, we apply these results to get decay estimates for linear semigroup and then prove the nonlinear asymptotic stability of all $n$-vortex relative to radial perturbations. In Sect. 4 , we use the variational characterization of principal eigenvalues, and the maximum principle to prove parts (2) and (3) of Theorem 1.1. We identify the possible growth modes of perturbations in $n$-vortex, $|n| \geqq 2$. We also comment on how to adapt our method here to show nonlinear asymptotic stability of one vortices on the finite disc domain with given Dirichlet data as treated in [19] and [22]; see Theorem 5.2. In Sect. 5, we prove Theorem 1.2 using results in Sect. 4, as well as the Hamiltonian structure of (1.7).

\section{Preliminary Analysis}

We consider the Ginzburg-Landau equation:

$$
\begin{aligned}
u_{t} & =\Delta u+\left(1-|u|^{2}\right) u, \quad x \in \mathbb{R}^{2}, \\
\left.u\right|_{t=0} & =u_{0}(x),
\end{aligned}
$$

where $u: \mathbb{R}_{+}^{1} \times \mathbb{R}^{2} \rightarrow \mathbb{R}^{2}$, and $\Delta$ is the two dimensional Laplacian. It is known that (2.1) admits vortex solutions of the form:

$$
\Psi_{n}=U_{n}(r) e^{i n \theta}, \quad n= \pm 1, \pm 2, \ldots .
$$

The basic properties of $U_{n}(r)$ are [24]:

1) $U_{n}(r)$ is the unique solution to the ODE problem:

$$
\begin{aligned}
U_{r r}+\frac{1}{r} U_{r}-\frac{n^{2}}{r^{2}} U+\left(1-U^{2}\right) U & =0, \\
U(0)=0, \quad U^{\prime}(r)>0, \quad U(+\infty) & =1 .
\end{aligned}
$$

2) $U_{n}(r)$ has asymptotic behavior:

$$
U_{n}(r) \sim a r^{n}\left(1-\frac{r^{2}}{4 n+4}\right), \quad \text { as } r \rightarrow 0,
$$

where $a$ is a positive constant, and

$$
U_{n}(r) \sim 1-\frac{n^{2}}{2 r^{2}}, \quad \text { as } r \rightarrow \infty .
$$

We are interested in studying problem (2.1) with initial data:

$$
u_{0}(x)=\left(U_{n}(r)+v_{0}(r, \theta)\right) e^{i n \theta},
$$

where $(r, \theta)$ is the polar coordinate of $\mathbb{R}^{2}$, and $v_{0}(r, \theta)$ is a small perturbation in $L^{p}\left(\mathbb{R}^{2}\right)$, with $p>1$ to be specified. We remark that writing the perturbation as in (2.6) is technically convenient for our later analysis and has no loss of generality. To examine the evolution of perturbation $v_{0}(r, \theta) e^{i n \theta}$, we write $u$ as

$$
u(t, r, \theta)=\left(U_{n}(r)+v(t, r, \theta)\right) e^{i n \theta} .
$$


Substituting (2.7) into (2.1) and using Eq. (2.2), we derive the following equation for $v$ :

$$
\begin{aligned}
v_{t}= & \Delta v+\frac{2 n i}{r^{2}} v_{\theta}-\frac{n^{2}}{r^{2}} v-U_{n}^{2} v-U_{n}^{2} \bar{v}+\left(1-U_{n}^{2}\right) v \\
& -U_{n}|v|^{2}-\left(2 U_{n} \operatorname{Re}\{v\}+|v|^{2}\right) v,
\end{aligned}
$$

where $M v$ is the linear part and $N(v)$ is the nonlinear part. Later, we will write $v$ into its real and imaginary parts $(v=\alpha+i \beta)$, and will also use $M$ to denote the resulting linear operator. Some details in deriving (2.8) are:

$$
\begin{aligned}
\left(v e^{i n \theta}\right)_{t} & =\Delta\left(U_{n}(r) e^{i n \theta}\right)+\Delta\left(v e^{i n \theta}\right)+\left(1-\left|U_{n}+v\right|^{2}\right)\left(U_{n}+v\right) e^{i n \theta}, \\
v_{t} & =-\left(1-U_{n}^{2}\right) U_{n}+e^{-i n \theta} \Delta\left(v e^{i n \theta}\right)+\left(1-\left|U_{n}+v\right|^{2}\right)\left(U_{n}+v\right),
\end{aligned}
$$

where we have used

$$
e^{-i n \theta} \Delta\left(v e^{i n \theta}\right)=\Delta v+\frac{2 n i}{r^{2}} v_{\theta}-\frac{n^{2}}{r^{2}} v
$$

and

$$
\nabla \theta=\frac{1}{r}(-\sin \theta, \cos \theta)
$$

If we express $v$ in terms of its real and imaginary parts, $v=\alpha+i \beta$, Eq. (2.8) can be rewritten as the system:

$$
\begin{aligned}
& \alpha_{i}=\Delta \alpha-\frac{2 n}{r^{2}} \beta_{\theta}+\left(-\frac{n^{2}}{r^{2}}+1-3 U_{n}^{2}\right) \alpha-U_{n}\left(\alpha^{2}+\beta^{2}\right)-2 U_{n} \alpha^{2}-\left(\alpha^{2}+\beta^{2}\right) \alpha \\
& \beta_{t}=\Delta \beta+\frac{2 n}{r^{2}} \alpha_{\theta}+\left(-\frac{n^{2}}{r^{2}}+1-U_{n}^{2}\right) \beta-2 U_{n} \alpha \beta-\left(\alpha^{2}+\beta^{2}\right) \beta
\end{aligned}
$$

with initial data: $\left(\alpha_{0}(r, \theta), \beta_{0}(r, \theta)\right)$.

Remark 2.1. In the case of the dynamics of Schrödinger vortices, then we replace the left-hand side of (2.1) with - $i u_{t}$. Subsequently in (2.9), the left-hand side vector $\left(\alpha_{t}, \beta_{t}\right)^{T}$ is replaced by $-J_{0}\left(\alpha_{t}, \beta_{t}\right)^{T}$, where $J_{0}$ is the unit symplectic matrix $\left(\begin{array}{cc}0 & -1 \\ 1 & 0\end{array}\right)$.

We shall first consider the radial case, i.e., $\alpha_{0}=\alpha_{0}(r), \beta_{0}=\beta_{0}(r)$. For functions $\alpha=\alpha(r, t)$, and $\beta=\beta(r, t)$, the system (2.9) reduces to:

$$
\begin{aligned}
& \alpha_{t}=\mathscr{L}_{1}^{(n)} \alpha-U_{n}\left(\alpha^{2}+\beta^{2}\right)-2 U_{n} \alpha^{2}-\left(\alpha^{2}+\beta^{2}\right) \alpha, \\
& \beta_{t}=\mathscr{L}_{2}^{(n)} \beta-2 U_{n} \alpha \beta-\left(\alpha^{2}+\beta^{2}\right) \beta,
\end{aligned}
$$

where

$$
\begin{aligned}
& \mathscr{L}_{1}^{(n)} \equiv \Delta+\left(-\frac{n^{2}}{r^{2}}+1-3 U_{n}^{2}(r)\right), \\
& \mathscr{L}_{2}^{(n)} \equiv \Delta+\left(-\frac{n^{2}}{r^{2}}+1-U_{n}^{2}(r)\right) .
\end{aligned}
$$

The operators have domain of definition $\mathscr{D}=\left\{u \in H^{2}\left(\mathbb{R}^{2}\right): r^{-2} u \in L^{2}\left(\mathbb{R}^{2}\right)\right\}$. We will estimate the semigroups generated by these two operators, and establish decay of solutions for system (2.10) in the coming two sections. Our results will hold for any $n$, so for ease of presentation we only consider $n=1$. We will replace $U_{n}$ by $U$, and abbreviate the operators in (2.11) and (2.12) into $\mathscr{L}_{i}, i=1,2$. 


\section{Gaussian Upper Bound for the Semigroup $e^{\mathscr{L}_{2} t}$}

In this section, we derive the Gaussian upper bound for the fundamental solution of the parabolic equation:

$$
u_{t}=\mathscr{L}_{2} u \text {. }
$$

Equation (3.1) is not in divergence form. The key idea is to make use of the vortex profile equation (2.3) to convert it into one. Let us verify the identity:

$$
\left(U^{-1} \mathscr{L}_{2} U\right) q=U^{-2} \nabla \cdot\left(U^{2} \nabla q\right),
$$

for any smooth function $q=q(r, \theta)$. We compute:

$$
\begin{aligned}
\Delta(U q) & =(\Delta U) q+U \Delta q+2 \nabla U \cdot \nabla q \\
& =-\left(1-U^{2}-\frac{1}{r^{2}}\right) U q+U \Delta q+2 \nabla U \cdot \nabla q,
\end{aligned}
$$

so

$$
\begin{aligned}
& \left(\Delta+1-U^{2}-\frac{1}{r^{2}}\right)(U q)=\mathscr{L}_{2}(U q) \\
& =U^{-1} \cdot\left(U^{2} \Delta q+2 U \nabla U \cdot \nabla q\right)=U^{-1} \nabla \cdot\left(U^{2} \nabla q\right),
\end{aligned}
$$

which is just (3.2). The semigroup $e^{\mathscr{L}_{2} t}$ is positivity preserving by parabolic maximum principle or by the Feynman-Kac formula [32]. If $U$ were not zero at $r=0$, then in view of (3.2), we could directly apply the results of Nash [23], Aronson [1], Osada [27] and others (see $[8,13,26]$ and references therein) to conclude that $U^{-1} \mathscr{L}_{2} U$ or $\mathscr{L}_{2}$ itself has pointwise upper and lower Gaussian bounds for their solution kernels. However, the fact that $U(0)=0$ makes the problem degenerate and prevents us from doing so. Actually there is no Gaussian lower bound for $\mathscr{L}_{2}$. This is easily seen; because for $r \sim 0, \mathscr{L}_{2} \sim\left(\Delta+1-\frac{1}{r^{2}}\right)$ which implies exponential decay of $e^{\mathscr{L}_{2} t}$ near $r=0$. To establish the Gaussian upper bound, we will introduce a smooth cutoff function $\eta$ compactly supported in a ball centered at zero. Outside this ball we use identity (3.2) and inside the ball we use the $-\frac{1}{r^{2}}$ term of $\mathscr{L}_{2}$ to help us overcome the degeneracy caused by $U(0)=0$. A careful construction of $\eta$ is necessary to piece the two parts together and achieve the Gaussian upper bound for the solution kernel of $\mathscr{L}_{2}$. We find it convenient to proceed along the line of proofs in Osada [27], who in turn followed the original ideas of Nash [23], Aronson [1], as well as Aronson and Serrin [2].

The properties of the function $\eta$ are summarized in:

Lemma 3.1. There exists a $C_{0}^{2}([0, \infty))$ function $\eta=\eta(r), r \geqq 0$, such that:

1) $\eta(r) \equiv 1$, if $r \in\left[0, r_{0}\right]$, where $r_{0} \in(0,1)$;

2) $\eta(r) \equiv 0$, if $r \geqq r_{1}$, where $r_{1} \in\left(r_{0}, 1\right)$, and $\eta>0$ if $r \in\left[0, r_{1}\right)$;

3) $0 \leqq \eta(r) \leqq 1, \eta_{r}(r) \leqq 0$, for all $r \geqq 0$;

4) for any $r \in \operatorname{supp}\{\eta\}$,

$$
2-2 U^{2}-\frac{2}{r^{2}}+\frac{4\left|\eta_{r}\right|^{2}}{\eta^{2}}+\frac{\Delta_{r} \eta}{\eta} \leqq-1
$$


and

$$
1-U^{2}-\frac{1}{r^{2}}+\frac{10\left|\eta_{r}\right|^{2}}{\eta^{2}}+\frac{\Delta_{r} \eta}{\eta} \leqq 0
$$

Proof. See appendix.

Let $p=p(t-s, x, y)$ be the fundamental solution of (3.1), which satisfies the semigroup property:

$$
p(t-s, x, y)=\int p(t-\tau, x, z) p(\tau-s, z, y) d z, \quad s<\tau<t .
$$

We then have:

Proposition 3.1. For any $s<t, x, y \in \mathbb{R}^{2}$, there is a positive constant $C$, depending only on the vortex profile $U$, such that:

$$
\begin{aligned}
\int_{R^{2}} p^{2}(t-s, x, y) d y & \leqq C(t-s)^{-1}, \\
\int_{R^{2}} p^{2}(t-s, x, y) d x & \leqq C(t-s)^{-1}, \\
p(t-s, x, y) & \leqq C(t-s)^{-1} .
\end{aligned}
$$

Proof. Note that (3.4) and (3.5) are similar, and (3.6) follows from (3.4) and (3.5) by the semigroup property. So we focus on the estimate (3.4). Next observe that we can, without loss of generality, set $s=0$, and $x=0$. It follows from (3.2) that:

$$
\begin{aligned}
q \mathscr{L}_{2} q & =q U\left(U^{-1} \mathscr{L}_{2} U\right)\left(U^{-1} q\right)=q U U^{-2} \nabla \cdot\left(U^{2} \nabla\left(U^{-1} q\right)\right) \\
& =U^{-1} q \nabla \cdot\left(U^{2} \nabla\left(U^{-1} q\right)\right) .
\end{aligned}
$$

Let

$$
E(t)=\int p^{2} \cdot\left(\eta^{2}+(1-\eta)^{2}\right) d y .
$$

With the notation, $\int=\int_{R^{2}} d y$, we have:

$$
\begin{aligned}
E_{t}= & \int 2 p p_{t}\left(\eta^{2}+(1-\eta)^{2}\right) d y=\int 2 p \mathscr{L}_{2} p\left(\eta^{2}+(1-\eta)^{2}\right) d y \\
= & \int 2\left(p \eta^{2}\right)\left(\Delta p+\left(1-U^{2}-\frac{1}{r^{2}}\right) p\right) \\
& \quad+\int 2 p(1-\eta)^{2}\left(\Delta p+\left(1-U^{2}-\frac{1}{r^{2}}\right) p\right) \\
\equiv & I+I I .
\end{aligned}
$$

Concerning $I$, our strategy is to use the dominance of $-r^{-2}$ for small $r$.

$$
\begin{aligned}
I= & -\int 2 \nabla\left(p \eta^{2}\right) \cdot \nabla p+2 \int(p \eta)^{2}\left(1-U^{2}-\frac{1}{r^{2}}\right) \\
= & -2 \int[\eta \nabla(p \eta)+p \eta \nabla \eta] \cdot \nabla p+2 \int p^{2} \eta^{2}\left(1-U^{2}-\frac{1}{r^{2}}\right) \\
= & -2 \int \nabla(p \eta) \cdot(\eta \nabla p+p \nabla \eta)+2 \int p \nabla(p \eta) \cdot \nabla \eta \\
& -2 \int \eta \nabla \eta \cdot p \nabla p+2 \int p^{2} \eta^{2}\left(1-U^{2}-\frac{1}{r^{2}}\right)
\end{aligned}
$$




$$
\begin{aligned}
= & -2 \int|\nabla(p \eta)|^{2}+2 \int p \nabla(p \eta) \cdot \nabla \eta \\
& +\int \nabla(\eta \nabla \eta) p^{2}+2 \int p^{2} \eta^{2}\left(1-U^{2}-\frac{1}{r^{2}}\right) \\
\leqq & -\int|\nabla(p \eta)|^{2}+\int\left[|\nabla|^{2} p^{2}+\nabla(\eta \nabla \eta) p^{2}+2 \eta^{2} p^{2}\left(1-U^{2}-\frac{1}{r^{2}}\right)\right] \\
= & -\int|\nabla(p \eta)|^{2}+\int p^{2} \eta^{2}\left(2-2 U^{2}-\frac{2}{r^{2}}+2 \frac{|\nabla \eta|^{2}}{\eta^{2}}+\frac{\Delta \eta}{\eta}\right)
\end{aligned}
$$

By the Nash inequality [23]: $\|u\|_{2}^{4} \leqq c_{0}^{-1}|| u\left\|_{1}^{2}|| \nabla u\right\|_{2}^{2}$,

$$
\int|\nabla(p \eta)|^{2} \geqq c_{0} \frac{\left(\int(p \eta)^{2}\right)^{2}}{\left(\int p \eta\right)^{2}} \geqq c_{0} \frac{\left(\int(p \eta)^{2}\right)^{2}}{\left(\int p\right)^{2}}=c_{0}\left(\int p^{2} \eta^{2}\right)^{2},
$$

where $c_{0}$ is a universal constant. Inequality (3.14) implies from (3.13) that:

$$
I \leqq-c_{0}\left(\int p^{2} \eta^{2}\right)^{2}+\int p^{2} \eta^{2}\left(2-2 U^{2}-\frac{2}{r^{2}}+\frac{2|\nabla \eta|^{2}}{\eta^{2}}+\frac{\Delta \eta}{\eta}\right)
$$

Now using (3.7), we have:

$$
\begin{aligned}
I I= & 2 \int(1-\eta)^{2} U^{-1} p \nabla \cdot\left(U^{2} \nabla\left(U^{-1} p\right)\right) \\
= & -2 \int U^{2} \nabla\left(U^{-1} p\right) \cdot \nabla\left((1-\eta)^{2} U^{-1} p\right) \\
= & -2 \int U^{2}\left((1-\eta) \nabla\left((1-\eta) U^{-1} p\right)+(1-\eta) U^{-1} p \nabla(1-\eta)\right) \cdot \nabla\left(U^{-1} p\right) \\
= & -2 \int U^{2}\left[\nabla\left((1-\eta) U^{-1} p\right)\left((1-\eta) \nabla\left(U^{-1} p\right)+U^{-1} p \nabla(1-\eta)\right)\right. \\
& \left.-U^{-1} p \nabla(1-\eta) \cdot \nabla\left((1-\eta) U^{-1} p\right)+(1-\eta) U^{-1} p \nabla(1-\eta) \cdot \nabla\left(U^{-1} p\right)\right] \\
= & -2 \int U^{2}\left[\left|\nabla\left((1-\eta) U^{-1} p\right)\right|^{2}-\left(U^{-1} p\right)^{2}|\nabla(1-\eta)|^{2}\right] \\
= & -2 \int U^{2}\left|\nabla\left((1-\eta) U^{-1} p\right)\right|^{2}+2 \int p^{2}|\nabla \eta|^{2} \\
\leqq & -2 c_{\eta} \int\left|\nabla\left((1-\eta) U^{-1} p\right)\right|^{2}+2 \int p^{2}|\nabla \eta|^{2}
\end{aligned}
$$

where here and below $c_{\eta}>0$ denotes a constant depending on $\eta$. Again, by Nash inequality, we have:

$$
\int\left|\nabla\left((1-\eta) U^{-1} p\right)\right|^{2} \geqq \mathcal{c}_{\eta}\left(\int(1-\eta)^{2} p^{2}\right)^{2}
$$

Inequalities (3.17) and (3.16) yield:

$$
I I \leqq-c_{\eta}\left(\int(1-\eta)^{2} p^{2}\right)^{2}+2 \int p^{2}|\nabla \eta|^{2}
$$


Combining (3.15) and (3.18), we get:

$$
\begin{aligned}
E_{t}=I & +I I \\
\leqq & \min \left(c_{0}, c_{\eta}\right)\left(\left(\int p^{2} \eta^{2}\right)^{2}+\left(\int(1-\eta)^{2} p^{2}\right)^{2}\right) \\
& +\int p^{2} \eta^{2}\left(2-2 U^{2}-\frac{2}{r^{2}}+4 \frac{|\nabla \eta|^{2}}{\eta^{2}}+\frac{\Delta \eta}{\eta}\right) \\
\leqq & -\frac{1}{2} \min \left(c_{0}, c_{\eta}\right)\left(\int p^{2}\left(\eta^{2}+(1-\eta)^{2}\right)\right)^{2} \\
& +\int p^{2} \eta^{2}\left(2-2 U^{2}-\frac{2}{r^{2}}+\frac{4|\nabla \eta|^{2}}{\eta^{2}}+\frac{\Delta \eta}{\eta}\right),
\end{aligned}
$$

or

$$
E_{t} \leqq-\frac{1}{2} \min \left(c_{0}, c_{\eta}\right) E^{2}+\int p^{2} \eta^{2}\left(2-2 U^{2}-\frac{2}{r^{2}}+\frac{4|\nabla \eta|^{2}}{\eta^{2}}+\frac{\Delta \eta}{\eta}\right) .
$$

By Lemma 3.1, we then have:

$$
E_{t} \leqq-\frac{1}{2} \min \left(c_{0}, c_{\eta}\right) E^{2}-\int p^{2} \eta^{2},
$$

which implies:

$$
E_{t} \leqq-\frac{1}{2} \min \left(c_{0}, c_{\eta}\right) E^{2}
$$

or

$$
E(t) \leqq \frac{C}{t}, \quad \forall t>0,
$$

where $C$ depends on $\eta$ and $U$. Inequality (3.4) follows. This completes the proof. Proposition 3.2. Let $r>0,(\sigma, x)$ be fixed. Let $v(y) \in L^{2}\left(\mathbb{R}^{2}\right) \cap L^{\infty}\left(\mathbb{R}^{2}\right)$ such that $v(y)=0$ if $|y-x|<r$. Suppose that $u(t, y)$ is a solution of the Cauchy problem of $\left(\partial_{t}-\mathscr{L}_{2}\right) u=0$ in $(\sigma, \infty) \times \mathbb{R}^{2}$ with initial value $u(\sigma, y)=v(y)$. Then for any $t, \sigma<t \leqq \sigma+r^{2}$, we have:

$$
|u(t, x)| \leqq C(t-\sigma)^{-\frac{1}{2}} \cdot \exp \left\{-C r^{2} /(t-\sigma)\right\}\|v\|_{2},
$$

with $C$ a positive constant.

Proof. Without loss of generality, we assume $(\sigma, x)=(0,0)$. For $0<s<t$, define:

$$
h(s, y)=-C_{1}|y|^{2} /(2 t-s),
$$

for some $C_{1}>0$ to be chosen. Consider the equation

and set

$$
u_{t}=\Delta u+\left(1-U^{2}(r)-\frac{1}{r^{2}}\right) u \equiv \mathscr{L}_{2} u,
$$

$$
m(r)=\eta^{2}+(1-\eta)^{2} .
$$

Multiplying both sides of $(3.25)$ by $m(r) u e^{2 h}$, integrating over $(0, \tau) \times \mathbb{R}^{2}$, we have:

$$
\int_{0}^{\tau} \int_{R^{2}} m(r) u e^{2 h} u_{s} d s d y=\int_{0}^{\tau} d s \int_{R^{2}} m(r) u e^{2 h}\left(\Delta u+\left(1-U^{2}-r^{-2}\right) u\right) d y .
$$


The left-hand side of (3.26) is:

$$
\left.\int_{R^{2}} \frac{1}{2} m(r) e^{2 h} u^{2} d y\right|_{s=0} ^{s=\tau}-\int_{0}^{\tau} \int_{R^{2}} e^{2 h} m u^{2} h_{s} d s d y .
$$

The right-hand side of (3.26) is:

$$
\begin{aligned}
& \int_{0}^{\tau} \int^{2} \eta^{2} u e^{2 h}\left(\Delta u+\left(1-U^{2}-r^{-2}\right) u\right) d y d s \\
& +\int_{0}^{\tau} \int(1-\eta)^{2} u e^{2 h}\left(\Delta u+\left(1-U^{2}-r^{-2}\right) u\right) d y d s \\
& =\int_{0}^{\tau} \int^{2} \eta^{2} u e^{2 h}\left(\Delta u+\left(1-U^{2}-r^{-2}\right) u\right) d y d s \\
& \quad+\int_{0}^{\tau} \int(1-\eta)^{2} e^{2 h}\left(U^{-1} u\right) \nabla\left(U^{2} \nabla\left(U^{-1} u\right)\right) d y d s \\
& =\int_{0}^{\tau} \int \eta^{2} u e^{2 h}\left(\Delta u+\left(1-U^{2}-r^{-2}\right) u\right) d y d s \\
& \quad-\int_{0}^{\tau} \int \nabla\left((1-\eta)^{2} e^{2 h}\left(U^{-1} u\right)\right) U^{2} \nabla\left(U^{-1} u\right) d y d s \\
& \equiv I+I I .
\end{aligned}
$$

We can rewrite the first term as:

$$
\begin{aligned}
I & =\int_{0}^{\tau} \int-\nabla\left(\eta^{2} u e^{2 h}\right) \cdot \nabla u+\eta^{2} u^{2} e^{2 h}\left(1-U^{2}-r^{-2}\right) \\
& =-\int_{0}^{\tau} \int \eta^{2} e^{2 h}|\nabla u|^{2}-\int_{0}^{\tau} \int u \nabla\left(\eta^{2} e^{2 h}\right) \cdot \nabla u+\int_{0}^{\tau} \int \eta^{2} u^{2} e^{2 h}\left(1-U^{2}-r^{-2}\right) \\
& =-\int_{0}^{\tau} \int \eta^{2} e^{2 h}|\nabla u|^{2}+\frac{1}{2} \iiint \Delta\left(\eta^{2} e^{2 h}\right) u^{2}+\int_{0}^{\tau} \int \eta^{2} u^{2} e^{2 h}\left(1-U^{2}-r^{-2}\right) .
\end{aligned}
$$

Using the Cauchy-Schwarz inequality, the second term can be estimated as follows:

$$
\begin{aligned}
I I= & -\int_{0}^{\tau} \int(1-\eta)^{2} U^{2} e^{2 h}\left|\nabla\left(U^{-1} u\right)\right|^{2} d y d s-\int_{0}^{\tau} \int U u \nabla\left((1-\eta)^{2} e^{2 h}\right) \cdot \nabla\left(U^{-1} u\right) d y d s \\
= & -\int_{0}^{\tau} \int(1-\eta)^{2} U^{2} e^{2 h}\left|\nabla\left(U^{-1} u\right)\right|^{2} d y d s-\int_{0}^{\tau} \int U u(1-\eta)^{2} 2 e^{2 h} \nabla h \cdot \nabla\left(U^{-1} u\right) d y d s \\
& \quad-\int_{0}^{\tau} \int U u e^{2 h} \nabla\left((1-\eta)^{2}\right) \cdot \nabla\left(U^{-1} u\right) d y d s \\
\leqq & -\int_{0}^{\tau} \int(1-\eta)^{2} U^{2} e^{2 h}\left|\nabla\left(U^{-1} u\right)\right|^{2} d y d s+\frac{1}{4} \int_{0}^{\tau} \int(1-\eta)^{2}\left|\nabla\left(U^{-1} u\right)\right|^{2} e^{2 h} U^{2} d y d s \\
& +8 \int_{0}^{\tau} \int(1-\eta)^{2} e^{2 h} u^{2}|\nabla h|^{2} d y d s+\frac{1}{4} \int_{0}^{\tau} \int(1-\eta)^{2} U^{2} e^{2 h}\left|\nabla\left(U^{-1} u\right)\right|^{2} d y d s \\
& +8 \int_{0}^{\tau} \int u^{2} e^{2 h}|\nabla \eta|^{2} d y d s .
\end{aligned}
$$




$$
\begin{aligned}
=- & \frac{1}{2} \int_{0}^{\tau} \int(1-\eta)^{2} U^{2} e^{2 h}\left|\nabla\left(U^{-1} u\right)\right|^{2} d y d s \\
& +8 \int_{0}^{\tau} \int e^{2 h} u^{2}|\nabla h|^{2} d y d s+8 \int_{0}^{\tau} \int u^{2} e^{2 h}|\nabla \eta|^{2} d y d s .
\end{aligned}
$$

It follows, using the properties of $\eta$ in Lemma 3.1, that

$$
\begin{aligned}
I+I I \leqq & \int_{0}^{\tau} \int\left[\frac{\Delta \eta}{\eta}+10\left|\frac{\nabla \eta}{\eta}\right|^{2}+\left(1-U^{2}-r^{-2}\right)\right] e^{2 h} \eta^{2} u^{2} d y d s \\
& +\int_{0}^{\tau} \int\left(|\nabla h|^{2} \eta^{2}+10|\nabla h|^{2}+\Delta h\right) e^{2 h} u^{2} d y d s \\
\leqq & \int_{0}^{\tau} \int\left(\Delta h+11|\nabla h|^{2}\right) e^{2 h} u^{2} d y d s .
\end{aligned}
$$

By (3.26),

$$
\begin{aligned}
\left.\frac{1}{2} \int m(r) e^{2 h} u^{2}\right|_{s=0} ^{s=\tau} & \leqq \int_{0}^{\tau} \int\left(m h_{s}+11|\nabla h|^{2}+\Delta h\right) u^{2} e^{2 h} d y d s \\
& \leqq \int_{0}^{\tau} \int\left(44 C_{1}\left(C_{1}-\frac{m}{44}\right) \frac{|y|^{2}}{2 t-s}-\frac{4 C_{1}}{2 t-s}\right) u^{2} e^{2 h} d y d s .
\end{aligned}
$$

Choose $C_{1}=\frac{1}{44} \min \left\{m(r): r \in \mathbb{R}^{+}\right\}$. Then (3.32) implies, since $v$ is supported where $|y|>r$, that

$$
\sup _{s \in[0, t]} \int_{4|y|^{2} \leqq t} e^{2 h} u^{2}(s, y) d y \leqq C \int_{|y|>r} m(r) e^{2 h} v^{2}(y) d y .
$$

For $(s, y)$ such that $s \in(0, t), 4|y|^{2} \leqq t, h(s, y) \geqq-\frac{C_{1}}{4}$; for $(s, y)$ such that $s \in(0, t)$, $|y|>r$ :

$$
h \leqq-\frac{C_{1} r^{2}}{2 t-s} \leqq-\frac{C_{1} r^{2}}{2 t} .
$$

Thus

$$
\sup _{s \in[0, t]} \int_{4|y|^{2} \leqq t} u^{2}(s, y) d y \leqq C e^{-\frac{C_{1} r^{2}}{t}} \int_{|y|>r} v^{2}(y) d y \leqq C e^{-\frac{C_{1} r^{2}}{t}}\|v\|_{2}^{2},
$$

and so

$$
\int_{0}^{t} \int_{4|y|^{2} \leqq t} u^{2}(s, y) d y \leqq C t e^{-\frac{C_{1} r^{2}}{t}}\|v\|_{2}^{2}
$$

By the local parabolic estimate (see Proposition 3.4 below):

$$
u(t, 0) \leqq C t^{-1}\left(\int_{0}^{t} \int_{4|y|^{2} \leqq t} u^{2} d y d s\right)^{\frac{1}{2}},
$$

for some $C>0$. It follows from (3.34) and (3.35) that

$$
u(t, 0) \leqq C t^{-\frac{1}{2}} e^{-C_{1} \frac{r^{2}}{t}}\|v\|_{2},
$$

for $t \in\left(0, r^{2}\right]$. The proof is complete. 
Theorem 3.1. Let $p(t, s ; x, y)$ be the fundamental solution of $u_{t}=\mathscr{L}_{2} u$. Then

$$
0<p(t-s ; x, y) \leqq C_{1}(t-s)^{-1} e^{-C_{2}|x-y|^{2} /(t-s)},
$$

for all $t>s, x, y$, where $C_{1}$ and $C_{2}$ are two positive constants depending only on $U$.

Proof. We follow the arguments of Aronson [1] or Osada [27], and include them here for the sake of completeness. First, if $t-s>r^{2}$, by Proposition 3.1:

$$
p(t, s ; x, y) \leqq C(t-s)^{-1} \leqq C(t-s)^{-1} e^{-\frac{|x-y|^{2}}{4(t-s)}} .
$$

We now focus on the case $t-s \leqq r^{2}$. As in the proof of Proposition 3.1, the pointwise bound (3.37) is obtained using the semigroup property of $p(t-s, x, y)$, (3.3). We first break the integration region in (3.3) into the regions $\{z:|z-x| \geqq r\}$ and $\{z:|z-x| \leqq r\}$, and apply the Cauchy-Schwarz inequality to obtain

$$
p(t-s ; x, y) \leqq J_{1}+J_{2},
$$

where

$$
J_{1}=\left(\int_{|z-x| \geqq r} p^{2}(\tau-s ; x, z) d z\right)^{\frac{1}{2}}\left(\int_{|z-x| \geqq r} p^{2}(t-\tau ; z, y) d z\right)^{\frac{1}{2}}
$$

and

$$
J_{2}=\left(\int_{|z-x| \leqq r} p^{2}(\tau-s ; x, z) d z\right)^{\frac{1}{2}}\left(\int_{|z-x| \leqq r} p^{2}(t-\tau ; z, y) d z\right)^{\frac{1}{2}},
$$

where $s<\tau<t$. We now show that for $t-s \leqq r^{2}$ :

$$
\int_{|y-x|>r} p^{2}(t-s ; x, y) d y \leqq C(t-s)^{-1} e^{-\frac{C r^{2}}{t-s}} .
$$

To this end, we consider:

$$
u(s, x)=\int_{|z-y|>r} p(s-\sigma ; x, z) p(t-\sigma ; y, z) d z,
$$

which is the solution of equation $u_{s}=\mathscr{L}_{2} u, s \geqq \sigma$ with initial data:

$$
u(\sigma, x)=0, \quad \text { if }|x-y|<r ; u(\sigma, x)=p(t-\sigma ; y, x), \quad \text { if }|x-y|>r .
$$

By Proposition 3.1, $u(\sigma, x) \in L^{2} \cap L^{\infty}\left(\mathbb{R}^{2}\right)$; and by Proposition 3.2:

$$
u(t, y)=\int_{|z-y|>r} p^{2}(t-\sigma ; y, z) d z \leqq C(t-\sigma)^{-\frac{1}{2}} e^{-\frac{C r^{2}}{t-\sigma}}\|u(\sigma, z)\|_{2},
$$

which implies (3.39) by Proposition 3.1. Similarly,

$$
\int_{|z-y|>r} p^{2}(t-\sigma ; y, z) d y \leqq C(t-\sigma)^{-1} e^{-\frac{\sigma r^{2}}{(t-\sigma)}} .
$$


Now set $r=\frac{|x-y|}{2}, \tau=\frac{(s+t)}{2}$ and assume $t-s \leqq r^{2}$. Using (3.39) and Proposition 3.1 , we get

$$
J_{1} \leqq C(t-s)^{-1} e^{-C \frac{x^{2}}{(t-\bar{t})}} \leqq C(t-s)^{-1} \exp \left\{-c \frac{|x-y|^{2}}{t-s}\right\} .
$$

For $J_{2}$, we see that $|z-x| \leqq r=\frac{|x-y|}{2}$ implies $|z-y| \geqq r$. Hence we have

$$
\begin{aligned}
J_{2} & \leqq\left(\int_{|z-x| \leqq r} p^{2}(s-\tau ; x, z) d z\right)^{\frac{1}{2}}\left(\int_{|z-x| \leqq r} p^{2}(t-\tau ; z, y) d z\right)^{\frac{1}{2}} \\
& \leqq\left(\int_{|z-y| \geqq r} p^{2}(s-\tau ; x, z) d z\right)^{\frac{1}{2}}\left(\int_{|z-y| \geqq r} p^{2}(t-\tau, z, y) d z\right)^{\frac{1}{2}}
\end{aligned}
$$

by (3.42) and Proposition 3.1

$$
\leqq C(t-s)^{-1} e^{-\frac{C|x-y|^{2}}{t-s}} \text {. }
$$

Thus (3.37) holds if $t-s \leqq r^{2}$. This completes the proof.

Finally, we outline the proof of the local parabolic estimate:

Proposition 3.3. Let $u$ be a solution to $u_{t}=\mathscr{L}_{2} u$ and

$$
Q=Q(y, \sigma, t)=\left\{x \in \mathbb{R}^{2}:|x-y|^{2}<(t-\sigma) / 4\right\} \times(\sigma, t) .
$$

Then there exists a constant $C$ independent of $u, \sigma, t$ and $y$ such that

$$
|u(t, y)| \leqq C(t-\sigma)^{-1}\left(\int_{Q} u^{2}\right)^{\frac{1}{2}} .
$$

Proof. In view of (3.2), and that by comparison $\mathscr{L}_{2}$ is below $\Delta$ near $r=0$, it is easy to check that the fundamental inequalities of Aronson and Serrin [2] (or Proposition 2.2 of Osada [27]) hold for operator $\mathscr{L}_{2}$. The rest follows from [2] on local properties of solutions of parabolic equations.

\section{Nonlinear Asymptotic Stability in the Radial Case}

In this section, we prove that any $n$-vortex solution is asymptotically stable under small radial perturbations (part (1) of Theorem 1.1). We will proceed with $n=1$; the proof in the general case is the same except for minor modifications. Let us consider the parabolic system:

$$
\begin{aligned}
& \alpha_{t}=\mathscr{L}_{1} \alpha-U\left(\alpha^{2}+\beta^{2}\right)-2 U \alpha^{2}-\left(\alpha^{2}+\beta^{2}\right) \alpha, \\
& \beta_{t}=\mathscr{L}_{2} \beta-2 U \alpha \beta-\left(\alpha^{2}+\beta^{2}\right) \beta,
\end{aligned}
$$

where

$$
\begin{aligned}
& \mathscr{L}_{1} \alpha=\Delta \alpha+\left(-\frac{1}{r^{2}}+1-3 U^{2}(r)\right) \alpha, \\
& \mathscr{L}_{2} \beta=\Delta \beta+\left(-\frac{1}{r^{2}}+1-U^{2}(r)\right) \beta .
\end{aligned}
$$


The initial data $\left(\alpha_{0}, \beta_{0}\right) \in\left(L^{p}\left(\mathbb{R}^{2}\right)\right)^{2}$, for some $p \in(1, \infty)$ to be specified. When $\left(\alpha_{0}, \beta_{0}\right)$ is radially symmetric, system (4.1)-(4.2) governs the dynamics of radial perturbations of the one-vortex solution. We will establish a decay result for mild solutions of (4.1)-(4.2) in $L^{p}$ spaces without assuming radial symmetry.

We first note that the semigroups $e^{i \mathscr{L}_{i}}, i=1,2$, are positivity preserving. Results of the last section imply that $e^{t \mathscr{L}_{2}}$ has a Gaussian upper bound, and so by a comparison argument, we have:

Proposition 4.1. The semigroup $e^{t_{2}}$ satisfies:

$$
\left\|e^{t \mathscr{L}_{2}} \varphi\right\|_{p} \leqq C\|\varphi\|_{p}, \quad \forall t \geqq 0, \quad \forall p \in[1,+\infty], \quad \forall \varphi \in L^{p}\left(\mathbb{R}^{2}\right),
$$

and

$$
\left\|e^{t \mathscr{L}_{2}} \varphi\right\|_{q} \leqq C t^{-\left(p^{-1}-q^{-1}\right)}\|\varphi\|_{p}, \quad \forall 1 \leqq p<q \leqq \infty,
$$

with $C>0$ independent of $p$.

The next step is to obtain an upper bound for $e^{t \mathscr{L}_{1}}$. Following the proof of Proposition 3.1, inequality (3.20), and writing $\mathscr{L}_{1}=\mathscr{L}_{2}-2 U(r)^{2}$, we find that

$$
E(t)=\int_{R^{2}} \Gamma^{2}\left(\eta^{2}+(1-\eta)^{2}\right),
$$

where $\Gamma$ is the fundamental solution of the equation $u_{t}=\mathscr{L}_{1} u$, satisfies the inequality:

$$
\begin{aligned}
E_{t} & \leqq-\frac{1}{2} c_{\eta} E^{2}-\int \Gamma^{2} \eta^{2}-2 \int \Gamma^{2} U(r)^{2}\left(\eta^{2}+(1-\eta)^{2}\right) \\
& \leqq-\frac{1}{2} c_{\eta} E^{2}-\int \Gamma^{2}\left[\eta^{2}+2 U(r)^{2}\left(\eta^{2}+(1-\eta)^{2}\right)\right]
\end{aligned}
$$

Since $\eta=1$ for $r \in\left[0, r_{0}\right]$, we have on this interval that

$$
\eta^{2}+2 U(r)^{2}\left(\eta^{2}+(1-\eta)^{2}\right) \geqq \eta^{2}=\left(\eta^{2}+(1-\eta)^{2}\right) .
$$

On the other hand, $r>r_{0}$, we have

$$
2 U(r)^{2}\left(\eta^{2}+(1-\eta)^{2}\right) \geqq 2 U\left(r_{0}\right)^{2}\left(\eta^{2}+(1-\eta)^{2}\right) .
$$

It follows that

$$
E_{t} \leqq-\frac{1}{2} c_{\eta} E^{2}-\min \left(1,2 U\left(r_{0}\right)^{2}\right) \int \Gamma^{2}\left(\eta^{2}+(1-\eta)^{2}\right) \equiv-c_{0} E^{2}-c_{1} E .
$$

Integrating (4.8) from zero to $t$, and using $E \rightarrow+\infty$, as $t \rightarrow 0^{+}$, we get

$$
E(t) \leqq c_{1} c_{0}^{-1} \frac{e^{-c_{1} t}}{1-e^{-c_{1} t}},
$$

which implies that

$$
E(t) \leqq C t^{-1} e^{-c_{1} t},
$$

for any $t>0$, where $C>0$ depends on $c_{0}$ and $c_{1}$. It follows that Proposition 3.1 holds for $\Gamma$ with $(t-s)^{-1} e^{-c_{1}(t-s)}$ replacing $(t-s)^{-1}$. We are ready to show 
Proposition 4.2. The semigroup $e^{t_{1}}$ satisfies:

$$
\left\|e^{t \mathscr{L}_{1}}\right\|_{p} \leqq C,
$$

for $C>0$ independent of $p \in[1,+\infty]$;

$$
\left\|e^{t \mathscr{L}_{1}}\right\|_{2} \leqq C e^{-\mathcal{C}_{1} t}
$$

and

$$
\left\|e^{t \mathscr{L}_{1}} \varphi\right\|_{q} \leqq C t^{-\left(p^{-1}-q^{-1}\right)} e^{-c_{1} t\left(p^{-1}-q^{-1}\right)}\|\varphi\|_{p}
$$

for any $1 \leqq p<q \leqq+\infty$.

Proof. First we deduce from $\Gamma(t, x, y) \leqq C t^{-1} e^{-c_{1} t}, \forall x, y, t>0$, that

$$
\left\|e^{t \mathscr{L}_{1}} \varphi\right\|_{\infty} \leqq C t^{-1} e^{-c_{1} t}\|\varphi\|_{1} .
$$

For any $u_{0} \in L^{p} \cap L^{\infty}, 1 \leqq p<\infty$, let $u_{0}=u_{0}^{+}-u_{0}^{-}$, where $u^{+}=\max \left(u_{0}, 0\right)$, $u_{0}^{-}=-\min \left(u_{0}, 0\right)$. Then $e^{t \mathscr{L}_{1}} u=e^{t \mathscr{L}_{1}} u_{0}^{+}-e^{t \mathscr{L}_{1}} u_{0}^{-}$. For any $t>0, e^{t \mathscr{L}_{1}} u_{0}^{ \pm}>0$ by strong maximum principle. The comparison principle says that

$$
e^{t \mathscr{L}_{1}} u_{0}^{ \pm} \leqq e^{t \mathscr{L}_{2}} u_{0}^{ \pm},
$$

for any $t, x$. It follows that

$$
\begin{aligned}
\left\|e^{t \mathscr{L}_{1}} u_{0}\right\|_{p} & \leqq\left\|e^{t \mathscr{L}_{1}} u_{0}^{+}\right\|_{p}+\left\|e^{t \mathscr{L}_{1}} u_{0}^{-}\right\|_{p} \\
& \leqq\left\|e^{t \mathscr{L}_{2}} u_{0}^{+}\right\|_{p}+\left\|e^{t \mathscr{L}_{2}} u_{0}^{+}\right\|_{p} \\
& \leqq 2 C\left\|u_{0}\right\|_{p},
\end{aligned}
$$

for any $p \in[1,+\infty]$. Interpolating (4.13) and (4.14) gives (4.12). Finally if we replace $\Gamma$ by the solution $u$ of equation $u_{t}=\mathscr{L}_{1} u$ in the proof of Proposition 3.1, and drop the terms $-\int|\nabla(u \eta)|^{2}$ and $-2 \int U^{2}\left|\nabla\left((1-\eta) U^{-1} u\right)\right|^{2}$, we obtain without using the Nash inequality:

$$
E_{t} \leqq-c_{1} E,
$$

which gives the $L^{2}$ bound (4.11). The proof is complete.

Remark 4.1. The estimate (4.11) may be true for any $p \in[1, \infty]$, however we will not pursue it here since (4.10) and (4.12) are sufficient for our stability proof.

Based on Proposition 4.1 and Proposition 4.2, we present

Theorem 4.1. Let us consider the system of integral equations corresponding to $(4.1)-(4.2)$ :

$$
\begin{aligned}
& \alpha=e^{t \mathscr{L}_{1}} \alpha_{0}-\int_{0}^{t} e^{(t-s) \mathscr{L}_{1}}\left[U\left(\alpha^{2}+\beta^{2}\right)+2 U \alpha^{2}+\alpha\left(\alpha^{2}+\beta^{2}\right)\right], \\
& \beta=e^{t \mathscr{L}_{2}} \beta_{0}-\int_{0}^{t} e^{(t-s) \mathscr{L}_{2}}\left[2 U \alpha \beta+\left(\alpha^{2}+\beta^{2}\right) \beta\right],
\end{aligned}
$$

with initial data $\left(\alpha_{0}, \beta_{0}\right) \in\left(L^{p} \cap L^{q}\left(\mathbb{R}^{2}\right)\right)^{2}$, where $p \in[3,6), q=\gamma^{-1} p, \gamma \in$ $\left(1+\frac{p}{3}, 3\right)$. Then there exists $\varepsilon>0$ depending only on $U, p, \gamma$, such that if 
$\max \left(\left\|\left(\alpha_{0}, \beta_{0}\right)\right\|_{p},\left\|\left(\alpha_{0}, \beta_{0}\right)\right\|_{q}\right) \leqq \varepsilon$, system (4.15)-(4.16) has unique mild solutions $(\alpha, \beta) \in C\left([0,+\infty) ;\left(L^{p}\left(R^{2}\right)\right)^{2}\right)$. Moreover, we have the decay estimates:

$$
\begin{aligned}
\|\alpha\|_{p}(t) & \leqq C(1+t)^{-(\gamma-1) p^{-1}}, \\
\|\beta\|_{p}(t) & \leqq C(1+t)^{-2(\gamma-1) p^{-1}},
\end{aligned}
$$

for all $t \geqq 0$, where $C=C(\varepsilon)>0$. In particular, (4.17) and (4.18) imply the asymptotic stability of the vortex solution $U(r) e^{ \pm i \theta}$ under small radial perturbations.

Analyzing with the same method the analogous parabolic system:

$$
\begin{aligned}
& \alpha_{t}=\mathscr{L}_{1}^{(n)} \alpha-U_{n}\left(\alpha^{2}+\beta^{2}\right)-2 U_{n} \alpha^{2}-\left(\alpha^{2}+\beta^{2}\right) \alpha, \\
& \beta_{t}=\mathscr{L}_{2}^{(n)} \beta-2 U_{n} \alpha \beta-\left(\alpha^{2}+\beta^{2}\right) \beta,
\end{aligned}
$$

where

$$
\begin{aligned}
& \mathscr{L}_{1}^{(n)} \alpha=\Delta_{x, y} \alpha+\left(-\frac{n^{2}}{r^{2}}+1-3 U_{n}^{2}(r)\right) \alpha, \\
& \mathscr{L}_{2}^{(n)} \beta=\Delta_{x, y} \beta+\left(-\frac{n^{2}}{r^{2}}+1-U_{n}^{2}(r)\right) \beta,
\end{aligned}
$$

we obtain:

Corollary 4.1. Any n-vortex solutions $U_{n}(r) e^{i n \theta}, n= \pm 1, \pm 2, \ldots$, are asymptotically stable with algebraic rates given by (4.17) and (4.18) under small radial perturbations in $L^{p} \cap L^{q}\left(\mathbb{R}^{2}\right)$.

Proof of Theorem 4.1. First we show that (4.15)-(4.16) has unique local solutions in $C\left(\left[0, T^{\star}\right) ;\left(L^{p}\left(R^{2}\right)\right)^{2}\right)$. Letting $\left(R_{1}, R_{2}\right)$ be the right-hand side of $(4.15)-(4.16)$, we estimate:

$$
\begin{aligned}
\left\|R_{1}\right\|_{p} \leqq & C\left\|\alpha_{0}\right\|_{p}+C \int_{0}^{t}(t-s)^{-1 / p} e^{-c_{1} p^{-1}(t-s)}\left\|3 \alpha^{2}+\beta^{2}\right\|_{p / 2} d s \\
& +C \int_{0}^{t} t^{-2 / p} e^{-2 c_{1} p^{-1}(t-s)}\left\|\alpha^{3}+\beta^{3}\right\|_{p / 3} d s \\
\leqq & C\left\|\alpha_{0}\right\|_{p}+C \int_{0}^{t}(t-s)^{-1 / p} e^{-c_{1} p^{-1}(t-s)}\left(\|\alpha\|_{p}^{2}+\|\beta\|_{p}^{2}\right) d s \\
& +C \int_{0}^{t}(t-s)^{-2 / p} e^{-2 c_{1} p^{-1}(t-s)}\left(\|\alpha\|_{p}^{3}+\|\beta\|_{p}^{3}\right) d s,
\end{aligned}
$$

so for $t \in[0, T], T>0$, we have

$$
\begin{aligned}
\sup _{0 \leqq t \leqq T}\left\|R_{1}\right\|_{p}(t) \equiv\left\|R_{1}\right\|_{p, \infty} \leqq & C\left\|\alpha_{0}\right\|_{p}+C T^{1-\frac{1}{p}}\left(\|\alpha\|_{p, \infty}^{2}+\|\beta\|_{p, \infty}^{2}\right) \\
& +C T^{1-\frac{2}{p}}\left(\|\alpha\|_{p, \infty}^{3}+\|\alpha\|_{p, \infty}^{3}\right) .
\end{aligned}
$$


Similarly,

$$
\begin{aligned}
\left\|R_{2}\right\|_{p} \leqq & C\left\|\beta_{0}\right\|_{p}+C \int_{0}^{t}\|\alpha \beta\|_{p / 2}(t-s)^{-1 / p} d s \\
& +C \int_{0}^{t}\left\|\alpha^{2} \beta\right\|_{p / 3}(t-s)^{-2 / p} d s+C \int_{0}^{t}\left\|\beta^{3}\right\|_{p / 3}(t-s)^{-2 / p} d s,
\end{aligned}
$$

which gives

$$
\begin{aligned}
\left\|R_{2}\right\|_{p, \infty} \leqq & C\left\|\beta_{0}\right\|_{p}+C\|\alpha\|_{p, \infty} \cdot\|\beta\|_{p, \infty} \sup _{t \in[0, T]} \int_{0}^{t}(t-s)^{-1 / p} d s \\
& +C\|\alpha\|_{p, \infty}^{2} \cdot\|\beta\|_{p, \infty} \sup _{t \in[0, T]} \int_{0}^{t}(t-s)^{-2 / p} d s \\
& +C\|\beta\|_{p, \infty}^{3} \sup _{t \in[0, T]} \int_{0}^{t}(t-s)^{-2 / p} d s \\
\leqq & C\left\|\beta_{0}\right\|_{p}+C T^{1-\frac{1}{p}}\|\alpha\|_{p, \infty} \cdot\|\beta\|_{p, \infty} \\
& +C T^{1-\frac{2}{p}}\|\alpha\|_{p, \infty}^{2} \cdot\|\beta\|_{p, \infty}+C T^{1-\frac{2}{p}}\|\beta\|_{p, \infty}^{3} .
\end{aligned}
$$

It follows from (4.23)-(4.24) that $\left(R_{1}, R_{2}\right)$ is a bounded map from $C([0, T]$; $\left.\left(L^{p}\left(R^{2}\right)\right)^{2}\right)$ into itself; moreover, if $T \leqq \delta=\delta\left(\left\|\left(\alpha_{0}, \beta_{0}\right)\right\|_{p}\right)$, then there is a unique solution $(\alpha, \beta) \in C\left([0, T] ;\left(L^{p}\left(R^{2}\right)\right)^{2}\right)$ by the contraction mapping principle. Such a solution can be continued to any $t<T^{\star}$, for some $T^{\star} \leqq+\infty$.

Next we proceed to derive the estimate of $\|(\alpha, \beta)\|_{p}(t)$, independent of $T$, where $t \in[0, T], T>\delta$. Let us define the norms:

$$
\begin{aligned}
& \|\| \alpha\left\|_{p} \equiv \sup _{t \in[0, T]}(1+t)^{a}\right\| \alpha \|_{p}(t), \\
& \|\beta\|\left\|_{p} \equiv \sup _{t \in[0, T]}(1+t)^{b}\right\| \beta \|_{p}(t),
\end{aligned}
$$

where $T \in\left(0, T^{\star}\right)$ and $a>0, b>0$, to be chosen. It follows from (4.15) that

$$
\begin{aligned}
\|\alpha\| \|_{p} \leqq & \sup _{t \in[0, T]}(1+t)^{a}\left\|e^{t \mathscr{L}_{1}} \alpha_{0}\right\|_{p} \\
& +\sup _{t \in[0, T]}(1+t)^{a} \int_{0}^{t}\left\|e^{(t-s)_{2} \mathscr{L}_{1}}\left(U\left(\alpha^{2}+\beta^{2}\right)+2 U \alpha^{2}+\left(\alpha^{2}+\beta^{2}\right) \alpha\right)\right\|_{p} \\
\leqq & \left.\max _{t \in[0, \delta]}(1+t)^{a}\left\|e^{t \mathscr{L}_{1}} \alpha_{0}\right\|_{p}, \sup _{t \in[\delta, T]}(1+t)^{a}\left\|e^{t \mathscr{L}_{1}} \alpha_{0}\right\|_{p}\right) \\
& +\sup _{t \in[0, T]}(1+t)^{a} \int_{0}^{t}(t-s)^{-1 / p} e^{-c_{1} p^{-1}(t-s)}\left\|3 \alpha^{2}+\beta^{2}\right\|_{p / 2} d s \\
& +\sup _{t \in[0, T]}(1+t)^{a} \int_{0}^{t}(t-s)^{-2 / p} e^{-2 c_{1} p^{-1}(t-s)}\left\|\alpha^{3}+\beta^{2} \alpha\right\|_{p / 3} d s
\end{aligned}
$$




$$
\begin{aligned}
& \leqq \max \left(C(1+\delta)^{a}\left\|\alpha_{0}\right\|_{p}, \sup _{t \in[\delta, T]}\left(t^{-1} e^{-c_{1} t}\right)^{\left(q^{-1}-p^{-1}\right)}(1+t)^{a}\left\|\alpha_{0}\right\|_{q}\right) \\
& +\sup _{t \in[0, T]}\left[( 1 + t ) ^ { a } \int _ { 0 } ^ { t } ( ( t - s ) ^ { - 1 } e ^ { - c _ { 1 } ( t - s ) } ) ^ { - 1 / p } \left(( 1 + s ) ^ { - 2 a } \left|\|\alpha \mid\|_{p}^{2}\right.\right.\right. \\
& \left.+(1+s)^{-2 b}\|\| \beta\|\|_{p}^{2}\right) d s \\
& +(1+t)^{a} \int_{0}^{t}\left((t-s)^{-1} e^{-c_{1}(t-s)}\right)^{-2 / p}(1+s)^{-3 a}\|\| \alpha^{2} \|\left.\right|_{p} ^{3} d s \\
& \left.\left.+(1+t)^{a} \int_{0}^{t}\left((t-s)^{-1} e^{-c_{1}(t-s)}\right)^{-2 / p}(1+s)^{-2 b-a}\left\|\beta^{2}\right\|\left\|_{p}^{2} \cdot\right\||| \alpha \mid \|_{p}\right) d s\right] \\
& \leqq C(q, p, a)\left(\left\|\alpha_{0}\right\|_{L^{p} \cap L^{q}}+\|\| \alpha\left\|_{p}^{2}+\right\|\|\beta\|_{p}^{2}+\left\||| \alpha\left|\left\|_{p}^{3}+\right\|\|\beta\|\right|_{p}^{2} \cdot\right\||\alpha| \|_{p}\right),
\end{aligned}
$$

under the condition

$$
a \leqq 2 b
$$

The integral term

$$
\sup _{t \in[0, T]}(1+t)^{a} \int_{0}^{t}(t-s)^{-1 / p} e^{-c_{1} p^{-1}(t-s)}(1+s)^{-2 b} d s
$$

appearing in (4.26) is uniformly bounded in $T$ under (4.27). Indeed,

$$
\begin{aligned}
& \sup _{t \in[0, \delta]}(1+t)^{a} \int_{0}^{t}(t-s)^{-1 / p} e^{-c_{1} p^{-1}(t-s)}(1+s)^{-2 b} d s \\
& \leqq(1+\delta)^{a} \int_{0}^{t}(t-s)^{-1 / p} d s=C(p, a) t^{1-2 / p} \leqq C(p, a) \delta^{1-2 / p}
\end{aligned}
$$

On the other hand, for $t \in(\delta, T]$, we have

$$
\begin{aligned}
\int_{0}^{t-\delta}(t-s)^{-1 / p} e^{-c_{1} p^{-1}(t-s)}(1+s)^{-2 b} d s & \leqq C(p) \int_{0}^{t-\delta} e^{-c_{1} p^{-1}(t-s)}(1+s)^{-2 b} d s \\
& \leqq C(p, b)(1+t)^{-2 b}
\end{aligned}
$$

and

$$
\begin{aligned}
\int_{t-\delta}^{t}(t-s)^{-1 / p} e^{-c_{1} p^{-1}(t-s)}(1+s)^{-2 b} d s & \leqq(1+t-\delta)^{-2 b} \int_{t-\delta}^{t}(t-s)^{-1 / p} d s \\
& \leqq(1+t-\delta)^{-2 b}(1-1 / p)^{-1} \delta^{1-1 / p}
\end{aligned}
$$


The other integral terms in (4.26) are analogous. Combining (4.28), (4.29), and (4.30), we arrive at (4.26). We obtain from (4.16) that

$$
\begin{aligned}
\|\beta\|_{p} \leqq & \max \left(C \sup _{t \in[0, \delta]}(1+t)^{b}\left\|\beta_{0}\right\|_{p}, \sup _{t \in[\delta, T]}(1+t)^{b} C t^{-\left(q^{-1}-p^{-1}\right)}\left\|\beta_{0}\right\|_{\bar{q}}\right) \\
& +C \sup _{t \in[0, T]}(1+t)^{b} \int_{0}^{t}(t-s)^{-(\gamma-1) p^{-1}}\|\alpha \beta\|_{p / \gamma} d s \\
& +C \sup _{t \in[0, T]}(1+t)^{b} \int_{0}^{t}(t-s)^{-2 p^{-1}}\left(\left\|\alpha^{2} \beta\right\|_{p / 3}+\left\|\beta^{3}\right\|_{p / 3}\right) d s .
\end{aligned}
$$

We choose

$$
q^{-1}=b+p^{-1}
$$

and note that

$$
\begin{aligned}
\mid \alpha \beta \beta \|_{p / \gamma} \leqq\left(\int|\alpha|^{p / \gamma}|\beta|^{p / \gamma}\right)^{\gamma / p} & \leqq\left(\int|\alpha|^{p /(\gamma-1)}\right)^{(\gamma-1) / p}\left(\int|\beta|^{p}\right)^{1 / p} \\
& =\|\alpha\|_{p /(\gamma-1)} \cdot\|\beta\|_{p}
\end{aligned}
$$

Now (4.31) gives

$$
\begin{aligned}
\|\beta\|_{p} \leqq & C\left\|\beta_{0}\right\|_{L^{p} \cap L^{q}}+C \sup _{t \in[0, T]}(1+t)^{b} \int_{0}^{t}(t-s)^{-(\gamma-1) p^{-1}}\|\alpha\|_{p /(\gamma-1)} \cdot\|\beta\|_{p} d s \\
& +C \sup _{t \in[0, T]}(1+t)^{b} \int_{0}^{t}(t-s)^{-2 p^{-1}}\left(\|\alpha\|_{p}^{2} \cdot\|\beta\|_{p}+\|\beta\|_{p}^{3}\right) d s .
\end{aligned}
$$

Then if

$$
q^{-1}>(\gamma-1) p^{-1}
$$

by (4.15) again

$$
\begin{aligned}
& \|\alpha\|_{p /(\gamma-1)}(t) \leqq C t^{-\left(q^{-1}-(\gamma-1) p^{-1}\right)} e^{-c_{1} t\left(q^{-1}-(\gamma-1) p^{-1}\right)}\left\|\alpha_{0}\right\|_{q} \\
& \quad+\int_{0}^{t} \| e^{(t-s) \mathscr{L}_{1}}\left(U\left(\alpha^{2}+\beta^{2}\right)+2 U \alpha^{2}+\left(\alpha^{2}+\beta^{2}\right) \alpha \|_{p /(\gamma-1)}\right. \\
& \leqq \\
& \leqq
\end{aligned}
$$




$$
\begin{aligned}
& +C \int_{0}^{t}\left((t-s)^{-1} e^{-c_{1}(t-s)}\right)^{3 p^{-1}-(\gamma-1) p^{-1}}\left(\|\alpha\|_{p}^{3}+\|\alpha\|_{p} \cdot\|\beta\|_{p}^{2}\right) \\
\equiv & C t^{-\left(q^{-1}-(\gamma-1) p^{-1}\right)} e^{-c_{1} t\left(q^{-1}-(\gamma-1) p^{-1}\right)}|| \alpha_{0} \|_{q}+I .
\end{aligned}
$$

Thus

$$
\sup _{t \in[0, T]}(1+t)^{2 b} I \leqq C\left(\left\||| \alpha\left|\left\|_{p}^{2}+\right\|\|\beta\|\right|_{p}^{2}+\right\|\|\alpha\|_{p}^{3}+\left.\||| \beta\|\right|_{p} ^{2} \cdot\|\alpha \mid\| \|_{p}\right),
$$

where we have used the integrals

$$
\begin{aligned}
& \int_{0}^{t}\left((t-s)^{-1} e^{-c_{1}(t-s)}\right)^{\left(2 p^{-1}-(\gamma-1) p^{-1}\right)}\left((1+s)^{-2 a}+(1+s)^{-2 b}\right) \\
& \quad \leqq C(1+t)^{-2 b}, \\
& \int_{0}^{t}\left((t-s)^{-1} e^{-c_{1}(t-s)}\right)^{\left(3 p^{-1}-(\gamma-1) p^{-1}\right)}\left((1+s)^{-3 a}+(1+s)^{-2 b-a}\right) \\
& \quad \leqq C(1+t)^{-2 b},
\end{aligned}
$$

where $C=C(a, b, p, \gamma)$ under the condition

$$
0<(3-\gamma) p^{-1}<1, \quad 0<(4-\gamma) p^{-1}<1 .
$$

Combining (4.34), (4.36), and (4.37), we get

$$
\begin{aligned}
\|\beta\|_{p} \leqq & C\left\|\beta_{0}\right\|_{L^{p} \cap L^{q}}+C\left\|\alpha_{0}\right\|_{q} \cdot\|\beta\|_{p} \sup _{t \in[0, T]}(1+t)^{b} \\
& \times \int_{0}^{t}(t-s)^{-(\gamma-1) p^{-1}} C\left(s^{-1} e^{-c_{1} s}\right)^{\left(q^{-1}-(\gamma-1) p^{-1}\right)} d s \\
& +\tilde{C} \sup _{t \in[0, T]}(1+t)^{b} \int_{0}^{t}(t-s)^{-(\gamma-1) p^{-1}}(1+s)^{-3 b} d s \\
& +C\|\alpha\|_{p}^{2} \cdot\left\|\beta|\||_{p} \sup _{t \in[0, T]}(1+t)^{b} \int_{0}^{t}(t-s)^{-2 p^{-1}}(1+s)^{-2 a-b} d s\right. \\
& +C\|\beta\| \|_{p}^{3} \sup _{t \in[0, T]}(1+t)^{b} \int_{0}^{t}(t-s)^{-2 p^{-1}}(1+s)^{-3 b} d s
\end{aligned}
$$

where

$$
\tilde{C}=C\left(\left|\left\|\alpha\left|\left\|_{p}^{2}+\right\|\right|\left|\beta\left\|_{p}^{2}+\right\|\right||\alpha|\right\|_{p}^{3}+\|\| \beta\|\|_{p}^{2} \cdot\|\| \alpha\|\|_{p}\right)\|\| \beta \|\left.\right|_{p} .\right.
$$

We optimize the decay rate by choosing

$$
\begin{gathered}
a=2 b, \\
b=(\gamma-1) p^{-1}, \\
1<(\gamma-1) p^{-1}+2 b .
\end{gathered}
$$


It follows from (4.43) and (4.44) that

$$
\gamma>1+p / 3, \quad 3 b>1,
$$

which implies that $2 a+b=5 b>1$. Now (4.40) requires that $\gamma<3$. Since $p \geqq 3$, (4.45) says that $\gamma>2$ and $3 \leqq p<6$. Thus given any $p \in[3,6)$, we pick $\gamma \in(1+p / 3,3),(a, b)$ according to $(4.42)$ and $(4.43), q^{-1}=b+p^{-1}=\gamma p^{-1}$. Then (4.35), (4.40) and (4.44) hold. It follows from (4.26) and (4.41) that

$$
\|\|(\alpha, \beta)\left\|_{p} \leqq C\right\|\left(\alpha_{0}, \beta_{0}\right) \|_{L^{p} \cap L^{q}}+f\left(\|\alpha\|_{p},\|\mid \beta\| \|_{p}\right),
$$

where $f=f(x, y)$ is a fourth degree polynomial containing no linear terms. Thus if $\left\|\left(\alpha_{0}, \beta_{0}\right)\right\|_{L^{p} \cap L^{q}}$ is sufficiently small, $\|(\alpha, \beta) \mid\|_{p}$ remains bounded for all time. The proof is complete.

\section{Linear Stability in the Nonradial Case}

In this section, we consider the evolution of general (nonradial) perturbations of vortex solutions, and prove part (2) of Theorem 1.1. We will see that, in contrast to the $|n|=1$ vortices, there is a potential for destabilizing $|n|>1$ vortices due to nonradial effects. This is in agreement with J. Neu's [24] numerical observations of the instability of higher $|n|$-vortices, in particular the splitting of a $n$-vortex $(|n| \geqq 2)$ into $n$ individual one-vortices under suitable perturbations.

The system governing the perturbation $v=\alpha+i \beta$ of an $n$-vortex solution $U_{n} e^{i n \theta}$ is

$$
\begin{aligned}
& \alpha_{t}=\mathscr{L}_{1}^{(n)} \alpha-\frac{2 n}{r^{2}} \beta_{\theta}-U_{n}\left(\alpha^{2}+\beta^{2}\right)-2 U_{n} \alpha^{2}-\left(\alpha^{2}+\beta^{2}\right) \alpha \\
& \beta_{t}=\mathscr{L}_{2}^{(n)} \beta+\frac{2 n}{r^{2}} \alpha_{\theta}-2 U_{n} \alpha \beta-\left(\alpha^{2}+\beta^{2}\right) \beta
\end{aligned}
$$

where

$$
\begin{aligned}
& \mathscr{L}_{1}^{(n)} \alpha=\Delta \alpha+\left(-\frac{n^{2}}{r^{2}}+1-3 U_{n}^{2}(r)\right) \alpha, \\
& \mathscr{L}_{2}^{(n)} \beta=\Delta \beta+\left(-\frac{n^{2}}{r^{2}}+1-U_{n}^{2}(r)\right) \beta
\end{aligned}
$$

Consider the linear part. In view of the $\theta$ independence of the coefficients, we expand into Fourier series:

$$
\begin{aligned}
& \alpha=\sum_{m \in Z} \alpha_{m} e^{i m \theta}, \\
& \beta=\sum_{m \in Z} \beta_{m} e^{i m \theta} .
\end{aligned}
$$

Then $\left(\alpha_{m}, \beta_{m}\right)$ satisfies

$$
\begin{aligned}
& \alpha_{m, t}=\Delta_{r} \alpha_{m}+\left(-\frac{n^{2}}{r^{2}}+1-3 U_{n}^{2}(r)\right) \alpha_{m}+\frac{1}{r^{2}}\left(-m^{2} \alpha_{m}-2 i n m \beta_{m}\right), \\
& \beta_{m, t}=\Delta_{r} \beta_{m}+\left(-\frac{n^{2}}{r^{2}}+1-U_{n}^{2}(r)\right) \beta_{m}+\frac{1}{r^{2}}\left(2 i n m \alpha_{m}-m^{2} \beta_{m}\right),
\end{aligned}
$$


or in vector notation:

$$
\begin{aligned}
\left(\begin{array}{l}
\alpha_{m} \\
\beta_{m}
\end{array}\right)_{t}= & \Delta_{r}\left(\begin{array}{l}
\alpha_{m} \\
\beta_{m}
\end{array}\right)+\left(\begin{array}{rr}
-\frac{n^{2}}{r^{2}}+1-3 U_{n}^{2}(r) & 0 \\
0 & -\frac{n^{2}}{r^{2}}+1-U_{n}^{2}(r)
\end{array}\right) \cdot\left(\begin{array}{l}
\alpha_{m} \\
\beta_{m}
\end{array}\right) \\
& +\frac{1}{r^{2}}\left(\begin{array}{rr}
-m^{2} & -2 i n m \\
2 i n m & -m^{2}
\end{array}\right) \cdot\left(\begin{array}{l}
\alpha_{m} \\
\beta_{m}
\end{array}\right),
\end{aligned}
$$

where $A_{r}$ is the two dimensional radial Laplacian. The operator formed by the first two terms on the right-hand side of (5.8) is the operator we have analyzed in the radial case. It is easy to show that this operator has continuous spectrum equal to $(-\infty, 0]$ and the Nash-Aronson estimates in Sect. 3 imply that the $L^{2}$ spectrum equals $(-\infty, 0]$. The "rotational terms" $r^{-2} \beta_{\theta}$ and $r^{-2} \alpha_{\theta}$ produce the matrix:

$$
\left(\begin{array}{cc}
-m^{2} & -2 i n m \\
2 i n m & -m^{2}
\end{array}\right)
$$

whose determinant is equal to $m^{2}\left(m^{2}-4 n^{2}\right)$. Therefore, the matrix (5.9) has positive eigenvalue if

$$
m \neq 0, \quad m^{2}<4 n^{2}
$$

and the possibility of instability exists. As $n$ increases, the number of potentially destabilizing modes increases.

In case $|n|=1$, only $m= \pm 1$ could be a source of linear instabilities. While if $m \neq \pm 1$, then (5.9) is nonpositive, so by our results in the radial case, such $\left(\alpha_{m}, \beta_{m}\right)$ would decay to zero with time in $L^{p}$ spaces. Let us consider $n=1$ and $m=1$, the other cases of $|n|=|m|=1$ are treated identically.

Let us transform (5.8) into a real coefficient system by first writing it as:

$$
\begin{aligned}
\left(\begin{array}{l}
\alpha_{1} \\
\beta_{1}
\end{array}\right)_{t}= & \left(\Delta_{r}+1-U^{2}(r)\right)\left(\begin{array}{l}
\alpha_{1} \\
\beta_{1}
\end{array}\right) \\
& +\frac{2}{r^{2}}\left(\begin{array}{rr}
-1 & -i \\
i & -1
\end{array}\right) \cdot\left(\begin{array}{l}
\alpha_{1} \\
\beta_{1}
\end{array}\right)+\left(\begin{array}{rr}
-2 U^{2} & 0 \\
0 & 0
\end{array}\right) \cdot\left(\begin{array}{l}
\alpha_{1} \\
\beta_{1}
\end{array}\right) .
\end{aligned}
$$

The matrix

$$
\left(\begin{array}{rr}
-1 & -i \\
i & -1
\end{array}\right)
$$

has eigenvalues $0,-2$, corresponding to eigenvectors $\frac{1}{\sqrt{2}}(i,-1)^{T}, \frac{1}{\sqrt{2}}(i, 1)^{T}$. Let us make the change of variables:

$$
\left(\begin{array}{l}
\alpha_{1} \\
\beta_{1}
\end{array}\right)=\frac{1}{\sqrt{2}}\left(\begin{array}{rr}
i & i \\
-1 & 1
\end{array}\right) \cdot\left(\begin{array}{l}
\gamma \\
\delta
\end{array}\right)
$$

then

$$
\left(\begin{array}{l}
\gamma \\
\delta
\end{array}\right)_{t}=\mathscr{L}\left(\begin{array}{l}
\gamma \\
\delta
\end{array}\right)
$$


where

$$
\mathscr{L}=\left(\Delta_{r}+1-U^{2}(r)\right) \operatorname{Id}+\frac{2}{r^{2}}\left(\begin{array}{rr}
0 & 0 \\
0 & -2
\end{array}\right)-U^{2}\left(\begin{array}{ll}
1 & 1 \\
1 & 1
\end{array}\right) .
$$

The following property of the one-vortex profile $U$ is very useful in analysis appearing later in this section:

Proposition 5.1. Let $U=U(r)$ be the one vortex profile. Then

$$
r^{-1} U(r)>U_{r}(r), \quad \forall r>0 .
$$

Moreover, the self-adjoint operator

$$
\mathscr{L}_{3} \equiv \Delta_{r}+\left(1-3 U^{2}(r)\right),
$$

defined on $H^{2}\left(\mathbb{R}^{2}\right)$ has spectrum $\sigma\left(\mathscr{L}_{3}\right)$ inside $\left(-\infty,-a_{0}\right)$, for some positive constant $a_{0}$.

Proof. Recall that $U(r)$ satisfies the equation:

$$
\begin{gathered}
U_{r r}+\frac{1}{r} U_{r}-\frac{1}{r^{2}} U+\left(1-U^{2}\right) U=0, \\
U(0)=0, \quad U(+\infty)=1, \quad U_{r}(r)>0,
\end{gathered}
$$

for any $r>0$. Differentiate (5.15) to $r$ and denote $U_{r}$ by $w$ to get:

$$
w_{r r}+\frac{1}{r} w_{r}-\frac{2}{r^{2}} w+\frac{2}{r^{3}} U+\left(1-3 U^{2}\right) w=0
$$

or

$$
w_{r r}+\frac{1}{r} w_{r}+\left(1-3 U^{2}\right) w=\frac{2}{r^{2}}\left(w-r^{-1} U\right) .
$$

Now letting $V=r^{-1} U$, we have from (5.15) that

$$
(r V)_{r r}+r^{-1}(r V)_{r}-r^{-1} V+\left(1-U^{2}\right) U=0,
$$

or

$$
r V_{r r}+2 V_{r}+V_{r}+r^{-1} V-r^{-1} V+\left(1-U^{2}\right) U=0,
$$

or

$$
V_{r r}+3 r^{-1} V_{r}=r^{-1}\left(U^{2}-1\right) U<0,
$$

for any $r>0$. We consider inequality (5.17) on $r \in\left[\varepsilon, r_{1}\right]$, where $\varepsilon \ll 1, r_{1} \gg 1$. For $r$ small, $U(r) \sim \operatorname{ar}\left(1-\frac{r^{2}}{8}+O\left(r^{4}\right)\right)$, for some constant $a>0$. Thus $V(r)$ is monotonely decreasing in $r$ if $r$ is small enough. With $\varepsilon$ sufficiently small, we see that $V(r)$ has to go through a local minimum if $V(r)$ increases with $r$ at all. In other words, there exists an interval $\left[r_{2}, r_{3}\right]$ strictly inside $\left[\varepsilon, r_{1}\right]$ such that $V$ has a minimum over $\left[r_{2}, r_{3}\right]$. However, inequality $(5.17)$ and strong maximum principle imply that $V(r)=$ const. for $r \in\left[r_{1}, r_{2}\right]$, or $U(r)=$ const. $r$. Therefore if $r \in\left[r_{2}, r_{3}\right]$, $U_{r r}=0, r^{-1} U_{r}-r^{-2} U=\left(r^{-1} U\right)_{r}=0$, but $\left(1-U^{2}\right) U>0$, contradicting (5.15). We conclude that

$$
V^{\prime}(r)=\left(\frac{U}{r}\right)_{r} \leqq 0,
$$


or $r U_{r} \leqq U(r)$, for any $r \geqq 0$. If $V^{\prime}\left(r_{4}\right)=0$, for some $r_{4}>0$, then (5.17) says that $V^{\prime \prime}\left(r_{4}\right)<0$, contradicting (5.18). Thus we have strict inequality in (5.18), and $r U_{r}<U(r)$, for any $r>0$.

Next we consider the spectrum of $\mathscr{L}_{3}$. By Weyl's theorem on the essential spectrum, we have that $\sigma_{\text {ess }}\left(\mathscr{L}_{3}\right)=(-\infty,-2]$. Moreover, $\mathscr{L}_{3}$ has a principal eigenvalue $\sigma_{1}$ and corresponding ground state eigenfunction $u_{1}=u_{1}(r)>0$ in $L^{2}\left(\mathbb{R}^{2}\right)$ such that

$$
\mathscr{L}_{3} u_{1}=\sigma_{1} u_{1}
$$

or what is the same:

$$
\Delta u_{1}+\left(1-3 U^{2}(r)\right) u_{1}=\sigma_{1} u_{1}
$$

for any $(x, y) \in \mathbb{R}^{2}$. By elliptic regularity $u_{1}$ is a smooth function. Similarly, we write $(5.16)$ as:

$$
\Delta w+\left(1-3 U^{2}(r)\right) w=\frac{2}{r^{2}}\left(w-r^{-1} U\right)<0 .
$$

Both $u_{1}$ and $w$ decay to zero as $r \rightarrow \infty$. Multiplying (5.20) by $w$, and integrating over $\mathbb{R}^{2}$, we get with integration by parts that:

$$
\int_{R^{2}} u_{1} \Delta w+\int_{R^{2}}\left(1-3 U^{2}\right) w u_{1}=\sigma_{1} \int_{R^{2}} u_{1} w,
$$

whose left-hand side is $\int_{R^{2}} \frac{2}{r^{2}}\left(U_{r}-r^{-1} U\right) u_{1}<0$. Noticing that $\int_{R^{2}} u_{1} w>0$, we infer that $\sigma_{1}<0$, and the proof of lemma is complete.

Proposition 5.2. The vector

$$
\frac{1}{2}\left(U_{r}+r^{-1} U(r), U_{r}-r^{-1} U\right) \equiv\left(\gamma_{0}, \delta_{0}\right)
$$

satisfies $\mathscr{L}\left(\gamma_{0}, \delta_{0}\right)^{T}=0$, for any $r=\left(x^{2}+y^{2}\right)^{\frac{1}{2}}$. However, $\left(\gamma_{0}, \delta_{0}\right) \notin L^{2}\left(\mathbb{R}^{2}\right)$ but is in $L^{p}\left(\mathbb{R}^{2}\right)$ for any $p>2$.

Remark. A mode of this type is frequently called a resonant state. It is known to influence the decay rate of the linear evolution operator generated by it. See, for example, [17].

Proof. It follows from (5.14) that $W \equiv \gamma+\delta$ satisfies:

$$
W_{t}=\Delta_{r} W+\left(1-3 U^{2}\right) W-\frac{4}{r^{2}} \delta .
$$

The pair $(W, \gamma)$ is the solution to the system:

$$
\begin{aligned}
W_{t} & =\Delta_{r} W+\left(1-3 U^{2}\right) W-\frac{4}{r^{2}}(W-\gamma), \\
\gamma_{t} & =\Delta_{r} \gamma+\left(1-U^{2}\right) \gamma-U^{2} W .
\end{aligned}
$$

Differentiating Eq. (5.15) to $r$ and letting $W_{0}=U_{r}$, we get:

$$
\Delta_{r} W_{0}+\left(1-3 U^{2}\right) W_{0}+\frac{2}{r^{2}}\left(r^{-1} U-U_{r}\right)=0 .
$$


Denoting

$$
\gamma_{0}=\frac{W_{0}}{2}+\frac{U}{2 r}, \quad \delta_{0}=\frac{W_{0}}{2}-\frac{U}{2 r}
$$

we have from (5.24) and (5.25) that

$$
\Delta_{r} W_{0}+\left(1-3 U^{2}\right) W_{0}-\frac{4}{r^{2}} \delta_{0}=\Delta_{r} W_{0}+\left(1-3 U^{2}\right) W_{0}-\frac{4}{r^{2}}\left(W_{0}-\gamma_{0}\right)=0 .
$$

Now using (5.24) and (5.15), we verify:

$$
\begin{aligned}
&\left(\Delta_{r}+\left(1-U^{2}\right)\right) \gamma_{0}-U^{2} W_{0} \\
&=\frac{1}{2}\left(\Delta_{r}+\left(1-U^{2}\right)\right)\left(W_{0}+\frac{U}{r}\right)-U^{2} W_{0} \\
&=\frac{1}{2}\left[\left(3 U^{2}-1\right) W_{0}+\frac{2}{r^{2}}\left(U_{r}-r^{-1} U\right)\right]+\frac{1}{2} \Delta_{r}\left(r^{-1} U\right) \\
&+\frac{1}{2}\left(1-U^{2}\right)\left(W_{0}+\frac{U}{r}\right)-U^{2} W_{0} \\
&=r^{-2}\left(U_{r}-r^{-1} U\right)+\frac{1}{2}\left(r^{-1} U_{r r}-2 r^{-2} U_{r}+2 r^{-3} U+r^{-2} U_{r}-r^{-3} U\right) \\
&+(2 r)^{-1}\left(1-U^{2}\right) U \\
&=r^{-2}\left(U_{r}-r^{-1} U\right)+\frac{1}{2}\left(r^{-1} \Delta_{r} U+r^{-3} U-2 r^{-2} U_{r}\right)+(2 r)^{-1}\left(1-U^{2}\right) U \\
&= r^{-2}\left(U_{r}-r^{-1} U\right)+\frac{1}{2} r^{-3} U+\frac{1}{2}\left(r^{-3} U-2 r^{-2} U_{r}\right)=0 .
\end{aligned}
$$

Thus $\left(\gamma_{0}, \delta_{0}\right)=\left(\frac{W_{0}}{2}+\frac{U}{2 r}, \frac{W_{0}}{2}-\frac{U}{2 r}\right)$ vanishes $\mathscr{L}$. Apparently, $\left(\gamma_{0}, \delta_{0}\right) \notin\left(L^{2}\left(\mathbb{R}^{2}\right)\right)^{2}$; however, belongs to $\left(L^{p}\left(\mathbb{R}^{2}\right)\right)^{2}$ if $p>2$. By Proposition 5.1, $\delta_{0}<0$, and $\gamma_{0}>0$, for any $r \geqq 0$. The proof is complete.

Proposition 5.3. Consider the self-adjoint operator $\mathscr{L}$ defined on

$$
\mathscr{D}=\left\{(\gamma, \delta) \in H^{2} \times H^{2}: r^{-2} \delta \in L^{2}\left(\mathbb{R}^{2}\right)\right\},
$$

Then the spectrum of $\mathscr{L}$ is equal to $(-\infty, 0]$.

Proof. By Weyl's essential spectrum theorem, $\sigma_{\text {ess }}(\mathscr{L})=(-\infty, 0]$. So we only need to prove that there is no positive eigenvalue. Suppose that $\sigma_{1}>0$ is the principal (the largest) eigenvalue of $\mathscr{L}$. By the variational characterization of the principal eigenvalue, we have:

$$
\sigma_{1}=\sup _{(\gamma, \delta) \in H^{1} \times H^{1},\|(\gamma, \delta)\|_{2}=1} Q(\gamma, \delta)
$$


where

$$
Q(a, b)=-\int_{R^{2}}\left(\gamma_{r}^{2}+\delta_{r}^{2}\right)+\int_{R^{2}}\left(1-2 U^{2}\right)\left(\gamma^{2}+\delta^{2}\right)-2 \int_{R^{2}} U^{2} \gamma \delta-4 \int_{R^{2}} r^{-2} \delta^{2} .
$$

Notice that the maximizer $\left(\gamma^{\star}, \delta^{\star}\right)$ of $Q$ must have $\delta^{\star}(r) \rightarrow 0$ as $r \rightarrow 0$ for $Q$ to stay finite. $\left(\gamma^{\star}, \delta^{\star}\right)$ is a classical solution for $r>0$. It is not hard to obtain $\delta^{\star}(r) \leqq O\left(r^{2}\right)$ by balancing terms in $\left(\mathscr{L}-\sigma_{1}\right)\left(\gamma^{\star}, \delta^{\star}\right)=0$. In fact, it follows from the $\delta^{\star}$ equation that

$$
\Delta_{r} \delta^{\star}-\frac{4}{r^{2}} \delta^{\star} \in L^{p}\left(\mathbb{R}^{2}\right)
$$

for any $p \in[2, \infty)$ due to $\left(\gamma^{\star}, \delta^{\star}\right) \in H^{1}\left(\mathbb{R}^{2}\right)$ and Sobolev imdedding. We can regard (5.29) as the $e^{2 i \theta}$ mode restriction of the two dimensional Laplacian. Hence, $\delta^{\star} \in W^{2, p}\left(\mathbb{R}^{2}\right), p>2$, and is imbedded into $C^{2+\varepsilon}, \varepsilon \in(0,1)$. Now we conclude by Taylor expanding $\delta^{\star}$ at zero, $r^{-2} \delta^{\star} \in L^{2}\left(\mathbb{R}^{2}\right)$ with (5.28), and (5.29). Thus $\left(\gamma^{\star}, \delta^{\star}\right) \in D(\mathscr{L})$. Thanks to the term $-2 \int_{R^{2}} U^{2} \gamma \delta$ and that $\int_{R^{2}}|\nabla f|^{2} \geqq\left.\int_{R^{2}}|\nabla| f\right|^{2}$ for any $f \in H^{1}\left(\mathbb{R}^{2}\right)$, we have

$$
Q\left(\gamma^{\star}, \delta^{\star}\right) \leqq Q\left(\left|\gamma^{\star}\right|,-\left|\delta^{\star}\right|\right)
$$

which implies that $\gamma^{\star}$ and $\delta^{\star}$ have opposite signs. That is either $\gamma^{\star} \geqq 0, \delta^{\star} \leqq 0$ or vice versa. We arrange $\gamma^{\star} \geqq 0$. Now forming the inner product in $L^{2}\left(\mathbb{R}^{2}\right)$ (denoted by $\left.(\cdot, \cdot)_{2}\right)$ of

$$
\left(\mathscr{L}-\sigma_{1}\right)\left(\gamma^{\star}, \delta^{\star}\right)=0
$$

with $\left(\gamma_{0}, \delta_{0}\right)$. Since $\sigma_{1}>0,\left(\gamma^{\star}, \delta^{\star}\right)$ decays to zero exponentially fast as $r \rightarrow \infty$. This can be seen as follows. The asymptotic behavior of $U$ at infinity (2.5) implies that $(5.30)$ is a weakly coupled elliptic system for large $r$ :

$$
\Delta_{r}\left(\begin{array}{l}
\gamma^{\star} \\
\delta^{\star}
\end{array}\right)-\left[\left(\begin{array}{rr}
1+\sigma_{1} & 1 \\
1 & 1+\sigma_{1}
\end{array}\right)+A(r)\right]\left(\begin{array}{l}
\gamma^{\star} \\
\delta^{\star}
\end{array}\right)=0,
$$

where $A(r)$ is a smooth 2 by 2 matrix in $r$, and $\|A\|_{\infty} \leqq O\left(r^{-2}\right)$. The matrix

$$
\left(\begin{array}{rr}
1+\sigma_{1} & 1 \\
1 & 1+\sigma_{1}
\end{array}\right)
$$

is positive definite and so can be diagonalized by a constant orthogonal matrix $Q_{1}$. Let

$$
\left(\gamma_{1}, \delta_{1}\right)^{T}=\left(\gamma^{*}, \delta^{*}\right)^{T} Q_{1}^{T}
$$

then $\left(\gamma_{1}, \delta_{1}\right)$ satisfies

$$
\Delta_{r}\left(\begin{array}{l}
\gamma_{1} \\
\delta_{1}
\end{array}\right)-\left[\left(\begin{array}{rr}
\lambda_{1} & 0 \\
0 & \lambda_{2}
\end{array}\right)+B(r)\right]\left(\begin{array}{l}
\gamma_{1} \\
\delta_{1}
\end{array}\right)=0,
$$

for $\lambda_{i}>0, i=1,2$, and a matrix $B=\left(b_{i j}\right),\|B\|_{\infty} \leqq O\left(r^{-2}\right)$. Letting $q=\gamma_{1}^{2}+\delta_{1}^{2}$, 
we have from (5.31):

$$
\begin{aligned}
\Delta_{r} q & =2 \gamma_{1} \Delta_{r} \gamma_{1}+2\left|\nabla \gamma_{1}\right|^{2}+2 \delta_{1} \Delta_{r} \delta_{1}+2\left|\nabla \delta_{1}\right|^{2} \\
& \geqq 2 \gamma_{1} \Delta_{r} \gamma_{1}+2 \delta_{1} \Delta_{r} \delta_{1} \\
& \geqq 2 \gamma_{1}\left(\lambda_{1} \gamma_{1}+b_{11}(r) \gamma_{1}+b_{12}(r) \delta_{1}\right)+2 \delta_{1}\left(\lambda_{2} \delta_{1}+b_{21}(r) \gamma_{1}+b_{22}(r) \delta_{1}\right) \\
& \geqq \lambda_{1} \gamma_{1}^{2}+\lambda_{2} \delta_{1}^{2} \geqq \min \left(\lambda_{1}, \lambda_{2}\right) q
\end{aligned}
$$

if $r$ is large enough depending on $\lambda_{i}, i=1,2$. Now it is easy to find a comparison function $\tilde{q}=e^{-\mu r}, \mu>0$ such that

$$
\left(\Delta_{r}-\min \left(\lambda_{1}, \lambda_{2}\right)\right) \tilde{q}=\left(\mu^{2}-\mu / r-\min \left(\lambda_{1}, \lambda_{2}\right)\right) e^{-\mu r} \leqq 0 .
$$

By comparison principle of scalar elliptic operators, we infer that

$$
q \leqq C e^{-\mu r}
$$

for some constant $C$ if $r$ is large enough. In other words, $\left(\gamma^{*}, \delta^{*}\right)$ decays exponentially fast as $r \rightarrow \infty$.

Thus we can perform integration by parts to get from $(5.30)$ :

$$
\left(\left(\gamma^{\star}, \delta^{\star}\right), \mathscr{L}\left(\gamma_{0}, \delta_{0}\right)\right)_{2}=\sigma_{1}\left(\left(\gamma^{\star}, \delta^{\star}\right),\left(\gamma_{0}, \delta_{0}\right)\right)_{2},
$$

whose right-hand side is strictly positive, and left-hand side is zero, impossible. Hence no positive eigenvalue exists for $\mathscr{L}$, and $\sigma(\mathscr{L})$ is $(-\infty, 0]$. This completes the proof.

We are now ready to prove part (2) of Theorem 1.1 .

Theorem 5.1. Consider the linearization of (5.1)-(5.2) for the plus or minus one vortex and let $\alpha+i \beta \in L^{2}\left(\mathbb{R}^{2}\right)$ be the solutions to the linearized system:

$$
\begin{aligned}
& \alpha_{t}=\mathscr{L}_{1}^{(1)} \alpha-\frac{2}{r^{2}} \beta_{\theta}, \\
& \beta_{t}=\mathscr{L}_{2}^{(1)} \beta+\frac{2}{r^{2}} \alpha_{\theta},
\end{aligned}
$$

with initial data $\left(\alpha_{0}+i \beta_{0}\right) \in L^{2}\left(\mathbb{R}^{2}\right)$. Then

$$
\|(\alpha, \beta)\|_{2}(t) \leqq\left\|\left(\alpha_{0}, \beta_{0}\right)\right\|_{2},
$$

for any $t \geqq 0$, i.e. the plus or minus one vortex is linearly dynamically stable in $L^{2}$ with respect to arbitrary (nonradial) $L^{2}$ perturbations. Moreover, the linearized operator in (5.34) denoted by $M$ is self-adjoint and nonpositive.

Proof. By our earlier discussion, we decompose $(\alpha, \beta)$ into the Fourier series:

$$
\begin{aligned}
& \alpha(t, r, \theta)=\sum_{j \in Z} \alpha_{j}(t, r) e^{i j \theta}, \\
& \beta(t, r, \theta)=\sum_{j \in Z} \beta_{j}(t, r) e^{i j \theta} .
\end{aligned}
$$


The problem is reduced to an analysis of the linear evolution operator on each invariant subspace corresponding to $e^{i j \theta}, j \in Z$. By a comparison argument with the reduced problem on each Fourier mode $j$, we find that the only source of instability of plus or minus vortex comes from $j= \pm 1$. This further reduces the problem to a study of semigroup $e^{t \mathscr{L}}$. Since $\mathscr{L}$ is dissipative (the real part of the $L^{2}$ inner product $\operatorname{Re}(\mathscr{L} f, f) \leqq 0)$, self-adjoint and densely defined, we have by the Lumer-Philips Theorem (Pazy [28]) that:

$$
\left\|e^{t \mathscr{L}}(f, g)\right\|_{2} \leqq\|(f, g)\|_{2} .
$$

The theorem now follows.

Remark. There is another way of proving Proposition 5.3 and Theorem 5.1. We take $m=n=1$ in (5.8), and make the change of variables: $a=i \alpha_{1}, b=\beta_{1}$. We obtain the following system with real coefficients for $(a, b)$ :

$$
\begin{aligned}
\left(\begin{array}{l}
a \\
b
\end{array}\right)_{t}= & \left(\begin{array}{rr}
\Delta_{r}+1-3 U^{2}(r) & 0 \\
0 & \Delta_{r}+1-U^{2}(r)
\end{array}\right)\left(\begin{array}{l}
a \\
b
\end{array}\right) \\
& +\frac{2}{r^{2}}\left(\begin{array}{cc}
-1 & 1 \\
1 & -1
\end{array}\right)\left(\begin{array}{l}
a \\
b
\end{array}\right) .
\end{aligned}
$$

Clearly, $(a, b)$ and $(\gamma, \delta)$ are related by a rotation:

$$
\left(\begin{array}{l}
a \\
b
\end{array}\right)=\frac{1}{\sqrt{2}}\left(\begin{array}{cc}
-1 & -1 \\
-1 & 1
\end{array}\right) \cdot\left(\begin{array}{l}
\gamma \\
\delta
\end{array}\right) .
$$

Then the zero resonance vector for the right-hand side operator in (5.36) is: $(a, b)=\left(U_{r}, U / r\right)$, whose two components are strictly positive. By the same variational argument, we can show that the right-hand side operator in (5.36) or equivalently $\mathscr{L}$ has spectrum $(-\infty, 0]$. The advantage of the $(a, b)$ variables is that we do not need Proposition 5.1 to prove Proposition 5.3 or Theorem 5.1. Also the zero resonance vector $\left(U_{r}, U / r\right)$ is simpler. The zero resonance vector comes from the translation invariance of the Ginzburg -Landau and Schrödinger evolutions (1.1) and (1.2).

We are however interested in obtaining a more refined understanding of the character of the zero point in the spectrum of the linearization about a vortex. We show, in our next result that the zero point is a pure resonance, i.e. there is no $L^{2}$ eigenfunction at zero energy. This provides an understanding of the resolvent of the linearized operator at zero energy which is required in obtaining decay estimates for the associated evolution operator. See, for example, [17]. To prove this result, it appears that the $(\gamma, \delta)$ variables are most expedient.

Proposition 5.4. Zero is a resonance and not an eigenvalue of $\mathscr{L}$.

Proof. The maximum principle is the main tool of the proof. Our method is a linear version of the sliding domain method (Berestycki and Nirenberg [6]) for nonlinear problems, and was used in an earlier work (Xin [37]).

Suppose $\left(\gamma^{\prime}, \delta^{\prime}\right) \in L^{2} \times L^{2}$ is an eigenfunction of $\mathscr{L}$ corresponding to zero, which is the principal eigenvalue by Proposition 5.3. By variational arguments, as in Proposition 5.3, $\gamma^{\prime}$ and $\delta^{\prime}$ have opposite signs and $\delta^{\prime}(r) \rightarrow 0$ as $r \rightarrow 0$. We choose $\delta^{\prime} \leqq 0$, for any $r \geqq 0$. The equations for $\left(W_{0}=\gamma_{0}+\delta_{0}, \delta_{0}\right)=\left(U_{r}, \delta_{0}\right)$ and 
$\left(W^{\prime} \equiv \gamma^{\prime}+\delta^{\prime}, \delta^{\prime}\right)$ are

$$
\begin{gathered}
\Delta_{r} W+\left(1-3 U^{2}\right) W-\frac{4}{r^{2}} \delta=0, \\
\Delta_{r} \delta+\left(1-U^{2}-4 r^{-2}\right) \delta-U^{2} W=0 .
\end{gathered}
$$

This implies, for $\lambda \in \mathbb{R}$, that the functions

$$
W_{\lambda} \equiv \lambda W_{0}-W^{\prime}, \quad \delta_{\lambda} \equiv \lambda \delta_{0}-\delta^{\prime},
$$

satisfy:

$$
\begin{aligned}
\Delta_{r} W_{\lambda}+\left(1-3 U^{2}\right) W_{\lambda} & =\frac{4}{r^{2}} \delta_{\lambda}, \\
\Delta_{r} \delta_{\lambda}+\left(1-U^{2}-4 r^{-2}\right) \delta_{\lambda} & =U^{2} W_{\lambda} .
\end{aligned}
$$

We first set $\lambda>0$. It follows from (5.40) that $W_{\lambda} \sim O\left(r^{-2} \delta_{\lambda}\right)$ due to $1-3 U^{2} \sim-2$ as $r \rightarrow \infty$. By (5.41) we have then: $\Delta_{r} \delta_{\lambda} \sim O\left(r^{-2} \delta_{\lambda}\right)$, which implies by direct integration that $\delta_{\lambda, r} \sim O\left(r^{-1} \delta_{\lambda}\right)$, and $\delta_{\lambda, r r} \sim O\left(r^{-2} \delta_{\lambda}\right)$. Going back to (5.40), we see that $W_{\lambda, r} \sim O\left(r^{-1} W_{\lambda}\right)$ and $W_{\lambda, r r} \sim O\left(r^{-2} W_{\lambda}\right)$ or $\Delta_{r} W_{\lambda}$ is a higher order decay term than $W_{\lambda}$. So by (5.40), $W_{\lambda} \sim-2 r^{-2} \delta_{\lambda}+$ h.o.t. Substituting this into (5.41) along with $1-U^{2} \sim r^{-2}+$ h.o.t., we obtain:

$$
\Delta_{r} \delta_{\lambda}+\left(-r^{-2}+\text { h.o.t. }\right) \delta_{\lambda}=0 .
$$

Since $\delta^{\prime} \in L^{2}\left(\mathbb{R}^{2}\right)$, for any given $\lambda>0$, there is $r_{0}=r_{0}(\lambda)>1$ such that $\delta_{\lambda}\left(r_{0}\right)<0$. We can choose $r_{0}(\lambda)$ large enough so that the above asymptotics become valid. By (5.42) and the fact that $\delta_{\lambda}(r) \rightarrow 0$ as $r \rightarrow \infty$, we deduce from the maximum principle that $\delta_{\lambda}$ has neither a nonnegative maximum nor a nonpositive minimum for any $r>r_{0}$. Therefore, $\delta_{\lambda}$ is negative for $r>r_{0}$, and monotonically increases to zero as $r \rightarrow \infty$. In other words, $\delta^{\prime}$ decays faster than $O\left(\delta_{0}\right)$. In particular, there exists $R_{0}$ such that:

$$
\delta_{\lambda}=\lambda \delta_{0}-\delta^{\prime}<0, \quad \text { if } r \geqq R_{0}, \lambda \geqq 1 .
$$

By making $R_{0}$ larger if necessary, we have $U \geqq 2 / 3$ for $r \geqq R_{0}$. We infer from (5.40) that

$$
\Delta_{r} W_{\lambda}+\left(1-3 U^{2}\right) W_{\lambda} \leqq 0,
$$

if $\lambda \geqq 1, r \geqq R_{0}$. For $r \in\left[0, R_{0}\right]$, there exists $\Lambda_{1}=\Lambda_{1}\left(R_{0}\right) \geqq 1$ such that if $\lambda \geqq \Lambda_{1}$, $W_{\lambda}(r)>0$ and $\delta_{\lambda}(r)<0$, for any $r \in\left[0, R_{0}\right]$. Thus if $\lambda \geqq \Lambda_{1},(5.44)$ and the maximum principle imply that $W_{\lambda}(r)>0$, any $r \geqq 0$. Also $\delta_{\lambda}<0, \forall r \in[0, \infty)$. Similarly, there is $\Lambda_{2} \geqq 1$, such that if $\lambda \leqq-\Lambda_{2}$, then $W_{\lambda}(r)<0, \delta_{\lambda}>0$, for any $r \geqq 0$. Define

$$
\mu \equiv \inf \left\{\lambda \in \mathbb{R}^{1} \mid W_{\lambda} \geqq 0 \text { and } \delta_{\lambda} \leqq 0, \forall r \in[0, \infty)\right\} .
$$

Then $\mu \in\left(-\Lambda_{2}, \Lambda_{1}\right), W_{\mu} \geqq 0, \delta_{\mu} \leqq 0, \forall r \in[0, \infty]$. Now suppose that $\mu=0$, then $\delta_{\mu}=-\delta^{\prime} \leqq 0$. However, as initially observed, $\delta^{\prime} \leqq 0$, and so $\delta^{\prime} \equiv 0$. By (5.38) and Proposition 5.1, we infer that $W^{\prime} \equiv 0$, or $\gamma^{\prime} \equiv 0$, contradicting the assumption that $\left(\gamma^{\prime}, \delta^{\prime}\right)$ is an eigenfunction. We deduce that $\mu>0$. 
With $\lambda=\mu$ in (5.40) and (5.41), $\delta_{\mu} \leqq 0, W_{\mu} \geqq 0$, strong maximum principle implies that either $W_{\mu}>0, \delta_{\mu}<0$, or $W_{\mu}=\delta_{\mu} \equiv 0$ for all $r \in[0, \infty)$. The latter implies $\mu \delta_{0} \equiv \delta^{\prime}$ and therefore $\delta_{0} \in L^{2}\left(\mathbb{R}^{2}\right)$, a contradiction.

Now suppose that there is a sequence $\left\{\lambda_{n}\right\}, \lambda_{n} \in(0, \mu), \lambda_{n} \rightarrow \mu$, as $n \rightarrow \infty$, such that

$$
\inf _{r \geqq 0} W_{\lambda_{n}}(r)<0,
$$

for each $\lambda_{n}$. Since $W_{\lambda_{n}}(r) \rightarrow 0$ as $r \rightarrow \infty$, there is a sequence $\left\{r_{n}\right\}$ such that

$$
W_{\lambda_{n}}\left(r_{n}\right)=\inf _{r \geqq 0} W_{\lambda_{n}}(r)<0 .
$$

If $\left\{r_{n}\right\}$ is unbounded, there is a subsequence, still denoted $\left\{r_{n}\right\}$, with $r_{n} \rightarrow \infty$. If $n$ is large enough, $r_{n}>R_{0}$, then evaluating

$$
\Delta_{r} W_{\lambda_{n}}\left(r_{n}\right)+\left(1-3 U^{2}\right) W_{\lambda_{n}}\left(r_{n}\right)=\frac{4}{r^{2}} \delta_{\dot{\lambda}_{n}}\left(r_{n}\right),
$$

at $r=r_{n} \gg 1$ yields

$$
\left(\Delta_{r} W_{\lambda_{n}}+\left(1-3 U^{2}\right) W_{\lambda_{n}}\right)\left(r_{n}\right) \geqq\left(1-3 U^{2}\left(r_{n}\right)\right) W_{\lambda_{n}}\left(r_{n}\right)>0,
$$

while

$$
\frac{4}{R_{N}^{2}}\left(\lambda_{n} \delta_{0}\left(r_{n}\right)-\delta^{\prime}\left(r_{n}\right)\right)<0,
$$

because $\lambda_{n} \rightarrow \mu>0$, and $\delta^{\prime}$ decays faster than $\delta_{0}$ at infinity as we have showed above by (5.42). We have a contradiction.

Therefore, $\left\{r_{n}\right\}$ is a bounded sequence, and there exists a subsequence, which we also denote $\left\{r_{n}\right\}$, along which we have $r_{n} \rightarrow r^{\star} \in[0, \infty)$. It follows that

$$
W_{\lambda_{n}}\left(r_{n}\right) \rightarrow W_{\mu}\left(r^{\star}\right) \leqq 0,
$$

as $n \rightarrow \infty$, contradicting $W_{\mu}(r)>0$, any $r \geqq 0$. This means that there is a number $\mu_{1} \in(0, \mu)$ such that

$$
\inf _{r \geqq 0} W_{\lambda}(r) \geqq 0, \quad \forall \lambda \in\left(\mu_{1}, \mu\right) .
$$

By minimality of $\mu$, we have

$$
\sup _{r \geqq 0} \delta_{\lambda}(r)>0, \quad \forall \lambda \in\left(\mu_{1}, \mu\right) .
$$

Therefore, there exists a sequence $\left\{\lambda_{n}^{\prime}\right\}, \lambda_{n}^{\prime} \in\left(\mu_{1}, \mu\right), \lambda_{n}^{\prime} \rightarrow \mu$, as $n \rightarrow \infty$, and a sequence of $\left\{r_{n}^{\prime}\right\}$ such that

$$
\delta_{\lambda_{n}}\left(r_{n}^{\prime}\right)=\sup _{r \geq 0} \delta_{\lambda_{n}}(r)>0 .
$$

If $\left\{r_{n}^{\prime}\right\}$ is unbounded, then $r_{n}^{\prime} \rightarrow \infty$, up to a subsequence still denoted the same. By Eq. (5.41) with $\lambda=\lambda_{n}$ :

$$
\Delta_{r} \delta_{\lambda_{n}}+\left(1-U^{2}-4 r^{-2}\right) \delta_{\lambda_{n}}=U^{2} W_{\lambda_{n}} \geqq 0 .
$$


If $r_{n}^{\prime} \gg 1$, then

$$
\begin{aligned}
1-U^{2}\left(r_{n}^{\prime}\right) & =\left(1-U\left(r_{n}^{\prime}\right)\right)\left(1+U\left(r_{n}^{\prime}\right)\right) \\
& =\left(\frac{1}{2\left(r_{n}^{\prime}\right)^{2}}+\text { h.o.t. }\right)\left(2-\frac{1}{2\left(r_{n}^{\prime}\right)^{2}}+\text { h.o.t. }\right) \\
& =\left(r_{n}^{\prime}\right)^{-2}+\text { h.o.t. }
\end{aligned}
$$

which shows that

$$
1-U^{2}\left(r_{n}^{\prime}\right)-4\left(r_{n}^{\prime}\right)^{-2}<0 .
$$

Evaluating (5.45) at $r=r_{n} \gg 1$ implies that the left-hand side is

$$
\leqq\left(1-U^{2}\left(r_{n}^{\prime}\right)-4\left(r_{n}^{\prime}\right)^{-2}\right) \delta_{\lambda_{n}}\left(r_{n}^{\prime}\right)<0,
$$

a contradiction. Finally, $\left\{r_{n}^{\prime}\right\}$ is bounded, and $r_{n} \rightarrow r^{\star \star} \in[0, \infty)$ along a subsequence still denoted the same. We have $\delta_{\mu}\left(r^{\star \star}\right) \geqq 0$, contradicting our early conclusion that $\delta_{\mu}<0$. Thus all roads from the assumption of zero being an $L^{2}$ eigenvalue lead to a contradiction. We conclude that zero is not an eigenvalue but rather a pure resonance. The proof is complete.

Finally, we comment on how to adapt our method to treat stability of one vortices on the disc of radius $R$, denoted by $B_{R}$. We will consider the plus one vortex to be specific. Let $u=U_{R}(r) e^{i \theta}$ be the plus one vortex solution on $B_{R}$, and consider perturbation of the form $v(t, r, \theta) e^{i \theta}$ such that $v(t, R, \theta)=0$. Going through the same derivation as before, we see that (5.1-5.2) hold for the real and imaginary parts of $v$, with $U_{n}$ replaced by $U_{R}$. We then decompose solutions into Fourier modes as in (5.5-5.6). For the radial part, or $m=0$, we follow the estimates in Proposition 3.1, however, they can be carried out directly on any solution $v$ of the linear equation $v_{t}=\mathscr{L}_{2} v$ since we can use the Poincare inequality instead of the Nash inequality thanks to the zero Dirichlet boundary condition of perturbation $v$ at $r=R$. The result is that $v$ decays to zero exponentially fast in the $L^{2}$ norm with a rate depending on $R$. Thus we only need to verify that the linearized operator $\mathscr{L}_{\mathscr{R}}$, which is just $\mathscr{L}$ in (5.14) with $U$ replaced by $U_{R}$ corresponding to the $m=1$ mode, has strictly negative spectrum. Using this strict negativity of $\mathscr{L}_{\mathscr{R}}$, we can prove:

Theorem 5.2. Let $U_{R}(r) e^{i n \theta}$ be a $|n|=1$ vortex on $B_{R}$ the disc of radius $R$. Let $u=\left(U_{R}(r)+v(t, r, \theta)\right) e^{i \theta}$, where $v$ is the perturbation satisfying $v(t, R, \theta)=0$ and $v(0, r, \theta) \in L^{2}\left(B_{R}\right)$. Then there exists constants $\gamma=\gamma(R)>0$, and $C=$ $C\left(\|v(0, r, \theta)\|_{2}\right)>0$, such that if $\|v(0, r, \theta)\|_{2}$ is small enough:

$$
\left\|u(t, r, \theta)-U_{R}(r) e^{i n \theta}\right\|_{2} \leqq C e^{-\gamma t},
$$

holds for all $t \geqq 0$. In other words, the one vortices are nonlinearly asymptotically stable with exponential rate.

Proof. We show that the operator $\mathscr{L}_{\mathscr{R}}$ has strictly negative spectrum. Since we are on a finite domain, $\mathscr{L}_{\mathscr{R}}$ has only discrete eigenvalues in the spectrum except for $-\infty$. Suppose that $\lambda_{1} \geqq 0$ is the leading eigenvalue corresponding to eigenvector $\left(v_{1}, v_{2}\right)$. By the variational principle, we can arrange so that $v_{1} \geqq 0, v_{2} \leqq 0$. Forming the $L^{2}$ inner product of $\left(\gamma_{0}, \delta_{0}\right)$ with

$$
\mathscr{L}_{R}\left(v_{1}, v_{2}\right)^{T}=\lambda_{1}\left(v_{1}, v_{2}\right)^{T},
$$


integrating by parts, and using the zero boundary condition on $\left(v_{1}, v_{2}\right)$, we get:

$$
\int_{r=R}\left(v_{1, r} \gamma_{0}+v_{2, r} \delta_{0}\right)=\lambda_{1}\left(\left(\gamma_{0}, \delta_{0}\right),\left(v_{1}, v_{2}\right)\right)_{2}
$$

On the other hand, $v_{1, r} \leqq 0, v_{2, r} \geqq 0$, at $r=R$, hence it follows that $\lambda_{1} \leqq 0$. So it is only possible that $\lambda_{1}=0$. Then the equation for $v_{1}$ is

$$
\left(\Delta_{r}+1-2 U_{R}^{2}(r)\right) v_{1}-U_{R}^{2}(r) v_{2}=0,
$$

or

$$
\left(\Delta_{r}-2 U_{R}^{2}(r)\right) v_{1}=-v_{1}+U_{R}^{2}(r) v_{2} \leqq 0,
$$

which implies via the strong maximum principle that either $v_{1} \equiv 0$ or $v_{1}>0$ if $r<R$. Similarly, $v_{2}$ satisfies the differential inequality:

$$
\left(\Delta_{r}-2 U_{R}^{2}(r)-\frac{4}{r^{2}}\right) v_{2}=-v_{2}+U_{R}^{2}(r) v_{1} \geqq 0,
$$

and so either $v_{2} \equiv 0$ or $v_{2}<0$ for $r<R$. Since $\left(v_{1}, v_{2}\right)$ is an eigenvector, one of its components is nonzero. Let us assume that $v_{1}>0$ (or $v_{2}<0$ ), $r<R$. Then by the Hopf lemma, $v_{1, r}<0$ (or $v_{2, r}>0$ ), at $r=R$. It follows that the left-hand side of (5.46) is strictly negative. We deduce a contradiction, and so $\lambda_{1}<0$. Since the spectrum is strictly negative, the linear evolution of the perturbation has to decay exponentially in time, and so is the nonlinear one as long as the initial perturbation is small enough. The proof is complete.

\section{Spectral Stability of the Schrödinger One-Vortex}

In this section, we show that the linearized operator for the Schrödinger one-vortex, $i \sigma M \equiv J M$, has spectrum equal to the imaginary axis. Therefore the Schrödinger one-vortex is spectrally stable. The perturbation $v(t, x)=(\alpha, \beta)^{T}$ to the Schrödinger one-vortex solution satisfies:

$$
\left(\begin{array}{l}
v \\
\bar{v}
\end{array}\right)_{t}=J M\left(\begin{array}{l}
v \\
\bar{v}
\end{array}\right)
$$

ignoring the nonlinear terms of $v$. By Weyl's theorem, the continuous spectrum of $J M$ is the entire imaginary axis, so we only need to show that there is no eigenvalue on the right half plane. Hamiltonian symmetry then ensures that there are no eigenvalues in the left half plane either.

Theorem 6.1. The operator $i \sigma M$ has $L^{2}$ spectrum equal to $i \mathbb{R}$.

Proof. The proof follows from a general result appearing in [29]. We present the argument in the current context. Suppose $J M \equiv i \sigma M$ has an eigenvalue $\lambda, \operatorname{Re}\{\lambda\}>$ 0 , corresponding to the eigenfunction $\psi$. Then

$$
J M \psi=\lambda \psi
$$

and so

$$
e^{J M t} \psi=e^{\lambda t} \psi
$$


Using skew symmetry of $J$ and symmetry of $M$, we have for any $u(t, x)$ satisfying $u_{t}=J M u$ :

$$
\begin{aligned}
\frac{d}{d t}(M u, u) & =\left(M \frac{d}{d t} u, u\right)+\left(M u, \frac{d}{d t} u\right) \\
& =(M J M u, u)+(M u, J M u) \\
& =(M J M u, u)+\left(M J^{\star} M u, u\right)=0,
\end{aligned}
$$

for any $u \in \mathscr{D}(M)$. It follows rather that

$$
0=\frac{d}{d t}\left(M e^{\lambda t} \psi, e^{\lambda t} \psi\right)=\frac{d}{d t} e^{(\lambda+\bar{\lambda}) t}(M \psi, \psi)
$$

which implies that

$$
(M \psi, \psi)=0 \text {. }
$$

Since $-M$ is a nonnegative self-adjoint operator,

$$
(-M \psi, \psi)=(\sqrt{-M} \psi, \sqrt{-M} \psi)=0,
$$

implying

$$
\sqrt{-M} \psi=0
$$

and so $M \psi=0$. In view of (6.48), we deduce that $\psi=0$, a contradiction, and the theorem is proved.

Remark 6.1. The previous theorem does not immediately imply linear dynamical stability of the Schrödinger one vortex. A key ingredient in controlling the time evolution of the linearized Schrödinger flow is an expansion of the resolvent of $M$ near the zero energy point. Proposition 5.4 is a component of this analysis, which we hope to pursue in future work. Note also that the operator $J M$ is not skew symmetric, so there is no immediate $L^{2}$ uniform bound.

\section{Appendix: Proof of Lemma 3.1}

Let us consider the $C_{0}^{\infty}$ function:

$$
\begin{aligned}
f(x) & =\left(a+\left(\tan ^{-1} \frac{\pi}{2} x\right)^{2}\right)^{-1}, \text { if } x \in[0,1] \\
& =0, \text { if } x \geqq 1
\end{aligned}
$$

where $a>0$ is a positive constant to be determined. We compute for $x \in[0,1)$ :

$$
f^{\prime}(x)=\frac{-\pi \tan ^{-1} \frac{\pi x}{2}}{\left(a+\left(\tan ^{-1} \frac{\pi x}{2}\right)^{2}\right)^{2}\left(1+\left(\frac{\pi}{2} x\right)^{2}\right)} \leqq 0,
$$


where equality holds at $x=0$, and

$$
\begin{aligned}
f^{\prime \prime}(x)= & \frac{4 \pi \cdot\left(\tan ^{-1} \frac{\pi x}{2}\right)^{2} \cdot \frac{\pi}{2}}{\left(a+\left(\tan ^{-1} \frac{\pi x}{2}\right)^{2}\right)^{3}\left(1+\left(\frac{\pi x}{2}\right)^{2}\right)^{2}}-\frac{\pi \cdot \frac{\pi}{2}}{\left(a+\left(\tan ^{-1} \frac{\pi x}{2}\right)^{2}\right)^{2}} \cdot \frac{1}{\left(1+\left(\frac{\pi}{2} x\right)^{2}\right)^{2}} \\
& +\frac{\pi \tan ^{-1} \frac{\pi x}{2}}{\left(a+\left(\tan ^{-1} \frac{\pi x}{2}\right)^{2}\right)^{2}} \cdot \frac{\frac{\pi^{2} x}{2}}{\left(1+\left(\frac{\pi}{2} x\right)^{2}\right)^{2}} \cdot
\end{aligned}
$$

Let $I=a+\left(\tan ^{-1} \frac{\pi x}{2}\right)^{2}, I I=1+\left(\frac{\pi x}{2}\right)^{2}$, then

$$
\begin{aligned}
f^{\prime \prime}(x)= & I^{-3} I^{-2}\left[2 \pi^{2}\left(\tan ^{-1} \frac{\pi}{2}\right)^{2}-\frac{\pi^{2}}{2}\left(a+\left(\tan ^{-1} \frac{\pi x}{2}\right)^{2}\right)\right. \\
& \left.+\frac{\pi^{3}}{2} x \tan ^{-1} \frac{\pi x}{2} \cdot\left(a+\left(\tan ^{-1} \frac{\pi x}{2}\right)^{2}\right)\right] \\
= & I^{-3} I^{-2} \cdot \frac{\pi^{2}}{2} \cdot\left[\pi x\left(\tan ^{-1} \frac{\pi x}{2}\right)^{3}+3\left(\tan ^{-1} \frac{\pi x}{2}\right)^{2}+\pi \operatorname{tax}\left(\tan ^{-1} \frac{\pi x}{2}\right)-a\right] \\
\equiv & I^{-3} I^{-2} \cdot \frac{\pi^{2}}{2} G(x) .
\end{aligned}
$$

We see that $G(x) \rightarrow+\infty$ as $x \rightarrow 1$, and for $x_{0}=\frac{2}{\pi \sqrt{3}}, x_{0}>\frac{1}{3}, \tan ^{-1} \frac{\pi x_{0}}{2}=\tan ^{-1} \frac{1}{\sqrt{3}}$ $=\frac{\pi}{6}$, so

$$
\begin{aligned}
G\left(x_{0}\right) & =\frac{2}{\sqrt{3}}\left(\frac{\pi}{6}\right)^{3}+3\left(\frac{\pi}{6}\right)^{2}+\left(\frac{2}{\sqrt{3}} \cdot \frac{\pi}{6}-1\right) a \\
& =\frac{2}{\sqrt{3}}\left(\frac{\pi}{6}\right)^{3}+3\left(\frac{\pi}{6}\right)^{2}+\left(\frac{\pi}{3 \sqrt{3}}-1\right) a<0
\end{aligned}
$$

if $a>\left(\frac{2}{\sqrt{3}}\left(\frac{\pi}{6}\right)^{3}+3\left(\frac{\pi}{6}\right)^{2}\right)\left(1-\frac{\pi}{3 \sqrt{3}}\right)^{-1} \equiv a^{*}$. Thus $\exists x^{*}=x^{*}(a), x^{\star} \in\left(\frac{1}{3}, 1\right)$, such that $G(x)>0$ if $x \in\left(x^{*}, 1\right)$ and $G\left(x^{*}\right)=0$. Let us consider:

$$
\frac{f^{\prime \prime}(x)}{f(x)}=\frac{\pi^{2}}{2} I^{-2} \cdot I^{-2} \cdot G(x)
$$

for $x \in\left(x^{*}, 1\right)$. Since

$$
x\left(\tan ^{-1} \frac{\pi x}{2}\right)^{3} \leqq \frac{\delta^{-1}}{2} x^{2}\left(\tan ^{-1} \frac{\pi x}{2}\right)^{2}+\delta\left(\tan ^{-1} \frac{\pi x}{2}\right)^{4}
$$

and

$$
x\left(\tan ^{-1} \frac{\pi x}{2}\right) \leqq \frac{\delta^{-1}}{2} x^{2}+\frac{\delta}{2}\left(\tan ^{-1} \frac{\pi x}{2}\right)^{2}
$$

for any constant $\delta>0$, we get

$$
G(x) \leqq \pi \delta\left(\tan ^{-1} \frac{\pi x}{2}\right)^{4}+\left(3+\frac{\pi \delta a}{2}+\frac{\pi \delta^{-1}}{2}\right)\left(\tan ^{-1} \frac{\pi x}{2}\right)^{2}+\frac{\delta^{-1} \pi a}{2}-a,
$$


and so

$$
\begin{aligned}
\frac{f^{\prime \prime}(x)}{f(x)} & \leqq \frac{\pi^{2}}{2} \cdot \frac{\pi \delta\left(\tan ^{-1} \frac{\pi x}{2}\right)^{4}+\left(3+\frac{\pi \delta a}{2}+\frac{\pi \delta^{-1}}{2}\right)\left(\tan ^{-1} \frac{\pi x}{2}\right)^{2}+\left(\frac{\pi \delta^{-1}}{2}-1\right) a}{a^{2}+2 a\left(\tan ^{-1} \frac{\pi x}{2}\right)^{2}+\left(\tan ^{-1} \frac{\pi x}{2}\right)^{4}} \\
& \leqq \frac{\pi^{2}}{2} \cdot\left(\pi \delta+\left(3+\frac{\pi \delta a}{2}+\frac{\pi \delta^{-1}}{2}\right)(2 a)^{-1}+\left|\frac{\pi \delta^{-1}}{2}-1\right| a^{-1}\right) \\
& =\frac{\pi^{2}}{2}\left(\pi \delta+\frac{3}{2 a}+\frac{\pi \delta}{4}+\frac{\pi \delta^{-1}}{4 a}+\left|\frac{\pi \delta^{-1}}{2}-1\right| a^{-1}\right) .
\end{aligned}
$$

It follows that $\forall \varepsilon>0, \exists \delta=\delta(\varepsilon), a=a(\delta)$, such that

$$
0<f^{\prime \prime}(x) / f(x) \leqq \varepsilon, \quad \forall x \in\left(x^{\star}, 1\right) .
$$

Obviously,

$$
\lim _{x \rightarrow x^{*}} \frac{f^{\prime \prime}(x)}{f(x)}=\lim _{x \rightarrow 1} \frac{f^{\prime \prime}(x)}{f(x)}=0 .
$$

We have now a function:

$$
\begin{aligned}
f(x) & \in C^{2}\left(\left[x^{*}, 1\right]\right), \quad f^{\prime}(x) \leqq 0, \quad f^{\prime \prime}(x) / f(x) \\
f^{\prime \prime}(x) & \geqq 0, \quad f(x) \geqq 0, \quad \forall x \in\left[x^{*}, 1\right] ; \quad f^{\prime \prime}\left(x^{*}\right)=0 .
\end{aligned}
$$

Let us define the function:

$$
g(x) \equiv \begin{cases}f(x), & x \in\left[x^{*}, 1\right], \\ 2 f\left(x^{*}\right)-f\left(2 x^{*}-x\right), & x \in\left[2 x^{*}-1, x^{*}\right] .\end{cases}
$$

It is easy to check that

$$
\begin{aligned}
& g(x) \in C^{2}\left(\left[2 x^{*}-1,1\right]\right), \quad g^{\prime \prime}\left(x^{*}\right)=0 ; \\
& g^{\prime}(x)<0, \quad \forall x \in\left(2 x^{*}-1,1\right) ; \\
& g^{\prime \prime}(x) / g(x)= \begin{cases}\frac{f^{\prime \prime}(x)}{f(x)}, & \forall x \in\left(x^{*}, 1\right), \\
\frac{-f^{\prime \prime}\left(2 x^{\star}-x\right)}{2 f\left(x^{*}\right)-f\left(2 x^{*}-x\right)}, & \forall x \in\left(2 x^{\star}-1, x^{\star}\right) .\end{cases}
\end{aligned}
$$

Notice that in (7.11), $f^{\prime \prime}\left(2 x^{*}-x\right) \geqq 0$. Moreover, $f^{\prime}(x) \leqq 0$ for $x \in\left[x^{\star}, 1\right]$ and $2 x^{*}-x \geqq x^{*}$ on $x \in\left(2 x^{*}-1, x^{*}\right)$ implies

$$
\begin{gathered}
f\left(2 x^{*}-x\right) \leqq f\left(x^{*}\right), \\
f\left(2 x^{*}-x\right)-2 f\left(x^{*}\right) \leqq-f\left(x^{*}\right)<0,
\end{gathered}
$$

on $x \in\left(2 x^{*}-1, x^{*}\right)$. It follows from $(7.13)$ that $g^{\prime \prime}(x) / g(x) \leqq 0$, for any $x \in\left(2 x^{*}-\right.$ $\left.1, x^{*}\right)$; while (7.12) gives

$$
2 f\left(x^{*}\right)-f\left(2 x^{*}-x\right) \geqq f\left(2 x^{*}-x\right) \geqq 0,
$$

or

$$
\left|\frac{f^{\prime}\left(2 x^{*}-x\right)}{2 f\left(x^{*}\right)-f\left(2 x^{*}-x\right)}\right| \leqq\left|\frac{f^{\prime}\left(2 x^{*}-x\right)}{f\left(2 x^{*}-x\right)}\right|,
$$


for $x \in\left[2 x^{*}-1, x^{*}\right]$. It follows that

$$
\begin{aligned}
& g(x) \in C^{2}\left(\left[2 x^{*}-1,1\right]\right), \\
& x^{*} \in\left(\frac{1}{3}, 1\right), \\
& g(x)>0, \quad \forall x \in\left[2 x^{*}-1,1\right), \\
& g^{(i)}(1)=0, \quad i=0,1,2 ; \\
& g^{(i)}\left(2 x^{*}-1\right)=0, \quad i=1,2, \\
& g\left(2 x^{*}-1\right)=2 f\left(x^{*}\right)>0, \\
& \left|g^{\prime}(x) / g(x)\right| \leqq \sup _{x \in\left[x^{*}, 1\right]}\left|f^{\prime}(x) / f(x)\right|, \quad \forall x \in\left[2 x^{*}-1,1\right], \\
& g^{\prime \prime}(x) / g(x) \leqq \varepsilon, \quad \forall x \in\left[2 x^{*}-1,1\right] .
\end{aligned}
$$

Now since $2 x^{*}-1>-1$, we extend

$$
g(x)=2 f\left(x^{*}\right), \quad \forall x \in\left[-1,2 x^{*}-1\right] ; \quad g(x)=0, \quad \forall x \in[1,+\infty)
$$

To summarize, we have

$$
\begin{aligned}
& g(x) \in C_{0}^{2}([-1,+\infty)), g(x)=2 f\left(x^{*}\right) \text { on }\left[-1,2 x^{*}-1\right) ; \\
& \frac{g^{\prime \prime}(x)}{g(x)} \leqq \varepsilon, \quad g^{\prime}(x) \leqq 0 \\
& \left|g^{\prime}(x) / g(x)\right| \leqq \sup _{x \in\left[x^{*}, 1\right]}\left|f^{\prime}(x) / f(x)\right|, \quad \forall x \in[-1,+\infty) ; \\
& g(x) \equiv 0, \quad \forall x \geqq 1
\end{aligned}
$$

We define

$$
\eta(x)=\left(2 f\left(x^{*}\right)\right)^{-1} g\left(\frac{x}{\alpha}-1\right), \quad \forall x \geqq 0,
$$

where $\alpha>0$ is a constant to be chosen. It follows from (7.18) that

$$
\begin{gathered}
\eta(x) \in C_{0}^{2}\left(\mathbb{R}_{+}^{1}\right), \quad 0 \leqq \eta \leqq 1, \\
\eta(x) \equiv 1, \quad \forall x \in\left[0,2 \alpha x^{*}\right], \\
\eta=0, \quad \forall x \geqq 2 \alpha .
\end{gathered}
$$

Let $r_{0}=2 \alpha x^{\star}, r_{1}=2 \alpha$. If $\alpha \in\left(0, \frac{1}{2}\right)$, then $r_{0}<r_{1}<1$. Moreover,

$$
\frac{\eta^{\prime}(x)}{\eta(x)}=\alpha^{-1} \frac{g^{\prime}\left(\frac{x}{\alpha}-1\right)}{g\left(\frac{x}{\alpha}-1\right)} .
$$


By our construction,

$$
\begin{aligned}
\left|g^{\prime}\left(\frac{x}{\alpha}-1\right) / g\left(\frac{x}{\alpha}-1\right)\right| & \leqq \sup _{x>0}\left|\frac{f^{\prime}(x)}{f(x)}\right| \\
& \leqq \sup _{x>0}\left|\frac{\pi \tan ^{-1} \frac{\pi x}{2}}{a+\left(\tan \frac{-1 \pi x}{2}\right)^{2}}\right| \leqq \frac{\pi}{2 \sqrt{a}} .
\end{aligned}
$$

So

$$
\left|\frac{\eta^{\prime}(x)}{\eta(x)}\right| \leqq \frac{2 \pi \alpha}{\sqrt{a}}, \quad \forall x>0
$$

On the other hand,

$$
\frac{\eta^{\prime \prime}(x)+\frac{1}{x} \eta^{\prime}(x)}{\eta(x)} \leqq \frac{\eta^{\prime \prime}(x)}{\eta(x)}=\frac{1}{\alpha^{2}} \frac{g^{\prime \prime}\left(\frac{x}{\alpha}-1\right)}{g\left(\frac{x}{\alpha}-1\right)} \leqq \frac{\varepsilon}{\alpha^{2}}, \quad \forall x>0
$$

Combining (7.20) and (7.21), we conclude that $\forall \varepsilon>0, \exists \eta(r) \in C_{0}^{2}\left(\mathbb{R}_{+}^{1}\right)$ such that: $\eta(r) \equiv 1$, if $r \in\left(0, r_{0}\right)$, where $r_{0} \in(0,1) ; \eta(r) \equiv 0$, if $r \geqq r_{1}$, where $r_{1} \in\left(r_{0}, 1\right)$; and $0 \leqq \eta(r) \leqq 1, \eta^{\prime}(r) \leqq 0, \forall r \geqq 0$. Moreover, we have

$$
\frac{\Delta \eta}{\eta}=\frac{\Delta_{r} \eta}{\eta} \leqq \frac{\varepsilon}{\alpha^{2}} ; \quad \frac{|\nabla \eta|}{\eta}=\frac{\left|\eta_{r}\right|}{\eta} \leqq \frac{\pi}{2 \alpha \sqrt{a}},
$$

for all $r \geqq 0$. For $r \in\left(0, r_{1}\right)$, that is on the support of $\eta$, we have

$$
\begin{gathered}
2-2 U^{2}-\frac{2}{r^{2}}+\frac{4|\nabla \eta|^{2}}{\eta^{2}}+\frac{A_{r} \eta}{\eta} \leqq 2-\frac{2}{r^{2}}+\frac{\pi^{2}}{\alpha^{2} a}+\frac{\varepsilon}{\alpha^{2}} \\
\leqq 2-\frac{2}{r_{1}^{2}}+\frac{\pi^{2}}{\alpha^{2} a}+\frac{\varepsilon}{\alpha^{2}}=2-\left(\frac{1}{2}-\varepsilon-\frac{\pi^{2}}{a}\right) \frac{1}{\alpha^{2}} .
\end{gathered}
$$

Similarly,

$$
1-U^{2}-\frac{1}{r^{2}}+\frac{10|\nabla \eta|^{2}}{\eta^{2}}+\frac{\Delta_{r} \eta}{\eta} \leqq 1-\left(\frac{1}{4}-\varepsilon-\frac{5 \pi^{2}}{2 a}\right) \frac{1}{\alpha^{2}}
$$

We take $\varepsilon=\frac{1}{8}$, then choose $a=a(\varepsilon)$ as in (7.8) and $a>40 \pi^{2}$; finally we make $\alpha$ small enough in (7.23). It follows that there exists $\alpha=\alpha_{0} \in\left(0, \frac{1}{2}\right)$ such that

$$
\begin{gathered}
2-2 U^{2}-\frac{2}{r^{2}}+\frac{4|\nabla \eta|^{2}}{\eta^{2}}+\frac{\Delta_{r} \eta}{\eta} \leqq 2-\frac{1}{4 \alpha^{2}} \leqq-1, \\
1-U^{2}-\frac{1}{r^{2}}+\frac{10|\nabla \eta|^{2}}{\eta^{2}}+\frac{\Delta_{r} \eta}{\eta} \leqq 0,
\end{gathered}
$$

for all $r \in\left(0, r_{1}=2 \alpha_{0}\right)$ which includes the support of $\eta$. This completes the proof of the lemma. 
Acknowledgements. Both authors wish to thank the Institut Mittag-Leffler for its hospitality and A. Kupiainen for organizing the workshop on Nonlinear PDE, Statistical Mechanics and Turbulence there in the Fall of 1994. M.I.W. also wishes to thank the Program in Applied and Computational Mathematics of Princeton University for its hospitality during the preparation of this paper. M.I.W. was supported in part by NSF grant DMS-9500997. J.X. was partially supported by NSF grant DMS-9302830, and the Swedish Natural Science Research Council (NFR) grant F-GF 10448-301 at the Institut Mittag-Leffler. He wishes to thank P. Deift, L. Friedlander, A. Jensen, D. Levermore, and Y. Pomeau for helpful conversations.

\section{References}

1. Aronson, D.G.: Bounds for fundamental solutions of a parabolic equation. Bull. Am. Math. Soc. 73, 890-896 (1967)

2. Aronson, D.G., Serrin, J.: Local behavior of solutions of quasilinear parabolic equations. Arch. Rational Mech. Anal. 25, 81-122 (1967)

3. Bauman, P., Chen, C., Phillips, D., Sternberg, P.: Vortex Annihilation in Nonlinear Heat Flow for Ginzburg-Landau Systems. European J. Appl. Math, to appear, 1995

4. Bethuel, T., Brezis, H., Helein, F.: Ginzburg-Landau Vortices. Boston: Birkhauser, 1994

5. Cycon, H.L., Froese, R.G., Kirsch, W., Simon, B.: Schrödinger Operators. Berlin-HeidelberNew York: Springer-Verlag Texts and Monographs in Physics, 1987

6. Berestycki, H., Nirenberg, L.: On the Method of Moving Planes and the Sliding Method. Bol. da Soc. Brasileria da Matematica 22, 1-37 (1991)

7. Creswick, R., Morrison, H.: Phys. Lett. A 76, 267 (1980)

8. Davies, E.B.: Heat Kernels and Spectral Theory. Cambridge: Cambridge Univ. Press, 1989

9. Demoulini, S., Stuart, D.: Gradient Flow of the Superconducting Ginzburg-Landau Functional on the Plane. Preprint, 1995

10. Donnelly, R.J.: Quantized Vortices in Helium II. Cambridge: Cambridge Univ. Press, 1991

11. E, W.: Dynamics of vortices in Ginzburg-Landau theories with applications to superconductivity. Physica D 77, 383-404 (1994)

12. Ercolani, N., Montgomery, R.: On the fluid approximation to a nonlinear Schrödinger equation. Phys. Lett. A 180, 402-408 (1993)

13. Fabes, E.B., Stroock, W.B.: A new proof of Moser's parabolic Harnack inequality using the old ideas of Nash. Arch. Rational Mech. Anal. 96, 327-338 (1986)

14. Fife, P., Peletier, L.A.: On the location of defects in stationary solutions of the GinzburgLandau equations on $\mathbb{R}^{2}$. Quart. Appl. Math, to appear

15. Frisch, T., Pomeau, Y., Rica, S.: Transition to Dissipation in a Model of Superflow. Phys. Rev. Lett. 69, No. 11, 1644-1647 (1992)

16. Jaffe, A., Taubes, C.: Vortices and Monopoles. Boston: Birkhäuser, 1980

17. Jensen, A., Kato, T.: Spectral properties of Schrödinger operators and time decay of wave functions. Duke Math. J. 46, 583-611 (1979)

18. Levermore, C.D., Xin, J.: Multidimensional Stability of Traveling Waves in a Bistable Reaction-Diffusion Equation, Part II. Comm. PDE 17(11 \& 12), 1901-1924 (1992)

19. Lieb, E.H., Loss, M.: Symmetry of the Ginzburg-Landau minimizer in a disc. Math. Res. Lett, 1, No. 8, 701-715 (1994)

20. Lin, F.-H.: Some Dynamical Properties of Ginzburg-Landau Vortices. Comm. Pure Appl. Math, to appear

21. Lin, F.-H.: A Remark on the Previous Paper "Some Dynamical Properties of Ginzburg-Landau Vortices". Preprint 1995

22. Mironescu, P.: On the Stability of Radial Solutions of the Ginzburg-Landau Equation. J. Func. Anal. 130, 334-344 (1995)

23. Nash, J.: Continuity of solutions of parabolic and elliptic equations. Am. J. Math. 80, 931-954 (1958)

24. Neu, J.: Vortices in the Complex Scalar Fields. Physica D 43, 385-406 (1990)

25. Newell, A.C.: Envelope equations. In: Nonlinear Wave Motion, Providence. RI: AMS, 1974, pp. $157-163$

26. Norris, J.R.: Long time behavior of heat flow: Global estimates and exact asymptotics. 1995, preprint 
27. Osada, H.: Diffusion processes with generators of generalized divergence form. J. Math. Kyoto Univ. (27-4), 597-619 (1987)

28. Pazy, A.: Semigroups of Linear Operators and Applications to PDE. Berlin-HeidelbergNew York: Springer Applied Math Sci. 44, 1983

29. Pego, R.L., Weinstein, M.I.: Eigenvalues, and solitary wave instabilities. Phil. Trans. R. Soc. Lond. A 340, 47-92 (1992)

30. Pismen, L., Rubinstein, J.: Dynamics of defects. In Nematics: Mathematical and Physical Aspects. J.M. Coron et. al. eds., Amsderdam: Kluwer Pubs, 1990

31. Rubinstein, J., Sternberg, P.: On the slow motion of vortices in the Ginzburg-Landau heat flow. SIAM J Math Anal, to appear

32. Simon, B.: Functional Integration and Quantum Physics. New York: Academic Press, 1979

33. Soffer, A., Weinstein, M.: Multichannel Scattering for Nonintegrable Equations, II, The Case of Anisotropic Potentials and Data. J. Diff. Eqs. 98, No. 2, 376-390 (1992)

34. Weinstein, W.: On the Vortex Solutions of Some Nonlinear Scalar Field Equations. Rocky Mountain J. Math, 21, No. 2, Spring, 821-826 (1991)

35. Weissler, F.: Existence and Non-existence of Global Solutions for a Semilinear Heat Equation. Isl J. Math. 38, No. 2, 1-2, 29-40 (1981)

36. Xin, J.: Multidimensional Stability of Traveling Waves in a Bistable Reaction-Diffusion Equation. Part I. Comm. PDE 17(11 \& 12), 1889-1899 (1992)

37. Xin, J.: Existence of Planar Flame Fronts in Convective-Diffusive Periodic Media. Arch. Rat. Mech. Anal. 121, 205-233 (1992)

38. Xin, J.: Existence of a Class of Symmetric Rotating Spiral Waves on Finite Dise Domains in Excitable Media. Indiana Univ. Math. J. 42, No. 4, Winter 1305-1337 (1993)

Communicated by A. Kupiainen 